\title{
Some New Applications of Russell's Principle to Infinite Dimensional Vibrating Systems
}

\author{
Scott W. Hansen, $\dagger$ \\ Department of Mathematics, Iowa State University \\ Ames, Iowa 50011 \\ shansen@iastate.edu \\ Marius Tucsnak ${ }^{\dagger}$ \\ Institut de Mathématiques de Bordeaux, Université de Bordeaux/CNRS/ Bordeaux INP \\ 351 Cours de Libération, 33405 Talence, France \\ Marius.Tucsnak@u-bordeaux.fr
}

Mathematics Subject Classification (2000): 35L10, 65M60, 93B05, 93B40, 93D15.

Key words: infinite dimensional systems, exact control, approximation, singular perturbation.

\section{Introduction}

The original version of Russell's principle says, roughly speaking that forwards and backwards exponential stabilizability implies exact controllability. The first application to systems governed by PDEs has been given by Russell himself in [29, Section 5], where he established the exact controllability for a wave equation with boundary control. This approach has been subsequently used to prove exact controllability for infinite dimensional systems by many authors, see for instance Chen [3] and Komornik [20]. An abstract version of this principle has been given in [3], in the case of bounded control operators. This version has been further generalized in Rebarber and Weiss [28] and Natarajan and Weiss [24]. More recently, a dual version Russell's principle, asserting that forward and backward detectability implies observability, came to the attention of control theorists. They developed, in particular, the concept of back and forth observers, which has been been proposed in Shim, Tanwani, and Ping [30] for finite dimensional, possibly nonlinear systems and in Ramdani, Tucsnak, and Weiss [27] for linear infinite dimensional systems (see also Ito, Ramdani and Tucsnak [17]).

The purpose of this work is to show that Russell's principle can be successfully adapted for a class of infinite dimensional systems involving perturbations and approximations. Intuitively, the exact controls constructed via this principle seem less "oscillating" then those obtained by inverting the Gramian (also designed, following Lions [22], by $H U M$

\footnotetext{
${ }^{*}$ The first author was supported in part by the National Science Foundation grant DMS-1312952.

${ }^{\dagger}$ Both authors acknowledge the support of the Institute of Mathematics and its Applications (IMA) during the special program 2015/2016 on control theory.
} 
method), which makes them more robust in view of perturbations and approximations. We focuss on two classes of applications.

The first one allows obtaining new results on a class of singular perturbation methods which have a geometric nature. More precisely, we want to understand the convergence of exact controls and of the associated state trajectories for a class of distributed control systems towards the corresponding objects for a boundary control system. The methodology is first developed in an abstract setting and then applied to families of systems governed by the string equation, with controls supported in a family of intervals shrinking towards an extremity of the string. As far as we know, the only papers to study this phenomena are Fabre [11, 9], Fabre and Puel [10] and Joly [19]. The main results in [11, 9, 10] provide sufficient conditions for the weak convergence of the minimal $L^{2}$ in time norm controls (often referred to as HUM controls) and of the corresponding trajectories towards those associated to the limiting control system. Joly in [19] investigated, in both the linear and nonlinear contexts, the strong convergence of solutions of the wave equation with locally distributed damping towards solutions of the wave equation with boundary damping. Unlike $[11,9,10]$, where minimal norm controls were considered, in this work we utilize controls generated using Russell's principle. This approach allows us to obtain a new abstract result that includes some cases of interest for the wave equation in one space dimension with stronger convergence properties. An important part of the effort in this part is devoted to obtaining wellposedness and observability estimates for the string equation which are independent of the size of the control interval (this requires an appropriate scaling of the control operator).

The second class of applications we are interested in is analyzing the approximation by finite dimensional systems, in particular giving error estimates, for exact controls for infinite dimensional vibrating systems. As a first step in this procedure we give a Russell's principle based construction of smooth controls for smooth initial states. We next consider the natural problem of approximating exact controls for infinite dimensional vibrating systems by controls associated to the projections of the considered systems on appropriate families of finite dimensional spaces. The work in this direction has been highly developed since the 90's, following a series of papers of Glowinski and Lions (see $[13,14]$ ) where algorithms to determine the minimal $L^{2}$-norm exact controls (sometimes called HUM controls) are provided. Several abnormalities presented in these works stand at the origin of a large number of articles in which a great variety of numerical methods are presented and analyzed (see, for instance, Zuazua et al. [34], [8] and the references therein). Much less is done concerning the rate of convergence of the approximations of these controls. In the case of HUM controls for the one dimensional wave equation, as far as we know, the only result in this direction has been obtained in Ervedoza and Zuazua [7]. In the last part of this work we describe, following Cîndea, Micu and Tucsnak [4], a Russell's principle based numerical method for computing exact controls for a class of infinite dimensional systems modelling elastic vibrations. Our main theoretical result gives the rate of convergence of our approximations to an exact control.

The remaining part of this work is organized as follows. In Section 2 we remind the derivation of Russell's principle in the particular case of systems governed by second order differential equations in a Hilbert space, with a bounded control operator. Some preliminary background results are provided in Section 3. Section 4 is devoted to an abstract singular perturbation result, which is given in Theorem 4.1. The proof that the $\varepsilon$-problems for the string with homogeneous Neumann boundary condition converge to the 
Neumann control problem (5.4)- (5.6) is given in Section 5 by checking that these systems fit the hypothesis of our general abstract result in Theorem 4.1. A similar procedure is used in Section 6 for the $\varepsilon$-problems for the string with homogeneous Dirichlet conditions and the limiting Dirichlet control problem (6.1)-(6.3). Finally, in Section 7 we describe a Russell's principle based strategy to approximate the exact controls for infinite dimensional vibrating systems using finite dimensional control systems and we provide some error estimates.

\section{The case of bounded input operators and construction of smooth con- trols}

For reader's convenience, we briefly recall below Russell's construction in the particular case of a second order linear differential equation in a Hilbert space and, to avoid technicalities, in the case of a bounded control operator. To this aim, let $\mathcal{H}$ be a Hilbert space. The inner product on $\mathcal{H}$ is denoted, for the remaining part of this paper, by $\langle\cdot, \cdot\rangle$ and the associated norm by $\|\cdot\|$. Let $\mathcal{U}$ be another Hilbert space. Throughout this work $\mathcal{H}$ and $\mathcal{U}$ will be identified with their duals and they will be used as pivot spaces when specifying the adjoints of various linear operators. Assume that $A_{0}: \mathcal{D}\left(A_{0}\right) \rightarrow \mathcal{H}$ is a self-adjoint, strictly positive operator with compact resolvents. Then, according to classical results, the operator $A_{0}$ is diagonalizable with an orthonormal basis $\left(\varphi_{k}\right)_{k \geqslant 1}$ of eigenvectors and the corresponding family of positive eigenvalues $\left(\lambda_{k}\right)_{k \geqslant 1}$ satisfies $\lim _{k \rightarrow \infty} \lambda_{k}=\infty$. Moreover, we have

$$
\mathcal{D}\left(A_{0}\right)=\left\{\left.z \in \mathcal{H}\left|\sum_{k \geqslant 1} \lambda_{k}^{2}\right|\left\langle z, \varphi_{k}\right\rangle\right|^{2}<\infty\right\},
$$

and

$$
A_{0} z=\sum_{k \geqslant 1} \lambda_{k}\left\langle z, \varphi_{k}\right\rangle \varphi_{k} \quad\left(z \in \mathcal{D}\left(A_{0}\right)\right) .
$$

For $\alpha \geqslant 0$ the operator $A_{0}^{\alpha}$ is defined by

$$
\mathcal{D}\left(A_{0}^{\alpha}\right)=\left\{\begin{array}{l|l}
z \in \mathcal{H} & \sum_{k \geqslant 1} \lambda_{k}^{2 \alpha}\left|\left\langle z, \varphi_{k}\right\rangle\right|^{2}<\infty
\end{array}\right\},
$$

and

$$
A_{0}^{\alpha} z=\sum_{k \geqslant 1} \lambda_{k}^{\alpha}\left\langle z, \varphi_{k}\right\rangle \varphi_{k} \quad\left(z \in \mathcal{D}\left(A_{0}^{\alpha}\right)\right) .
$$

For every $\alpha \geqslant 0$ we denote by $\mathcal{H}_{\alpha}$ the space $\mathcal{D}\left(A_{0}^{\alpha}\right)$ endowed with the inner product

$$
\langle\varphi, \psi\rangle_{\alpha}=\left\langle A_{0}^{\alpha} \varphi, A_{0}^{\alpha} \psi\right\rangle \quad\left(\varphi, \psi \in H_{\alpha}\right),
$$

and induced norm denoted by $\|\cdot\|_{\alpha}$. To be coherent with the notation above, we simply write $\langle\cdot, \cdot\rangle$ for $\langle\cdot, \cdot\rangle_{0}$ and $\|\cdot\|$ for $\|\cdot\|_{0}$. From the above facts it follows that for every $\alpha \geqslant 0$ the operator $A_{0}$ is a unitary operator from $\mathcal{H}_{\alpha+1}$ onto $\mathcal{H}_{\alpha}$ and $A_{0}$ is strictly positive on $\mathcal{H}_{\alpha}$.

Let $B_{0} \in \mathcal{L}(\mathcal{U}, \mathcal{H})$ be an input operator. Consider the system

$$
\ddot{q}(t)+A_{0} q(t)+B_{0} u(t)=0 \quad(t \geqslant 0),
$$




$$
q(0)=q_{0}, \dot{q}(0)=q_{1} .
$$

It is by now routine that for every $u \in L^{2}([0, \infty) ; \mathcal{U})$ the above equations admit a unique solution

$$
w \in C\left([0, \infty) ; \mathcal{H}_{\frac{1}{2}}\right) \cap C^{1}([0, \infty) ; \mathcal{H}),
$$

which satisfies, for every $u \in L^{2}([0, \infty) ; \mathcal{U})$ and $t \geqslant 0$, the energy estimate:

$$
\left.\frac{1}{2}\left(\|\dot{w}(0)\|^{2}+\|w(0)\|_{\frac{1}{2}}^{2}\right)-\frac{1}{2}\left(\|\dot{w}(t)\|^{2}+\|w(t)\|_{\frac{1}{2}}^{2}\right)=\int_{0}^{t}\left\langle u(\sigma), B_{0}^{*} \sigma\right)\right\rangle_{\mathcal{U}} \mathrm{d} \sigma .
$$

In other terms, equations (2.2)-(2.3) define a well-posed control system with input space $\mathcal{U}$ and state space $X=\mathcal{H}_{\frac{1}{2}} \times \mathcal{H}$.

The above system is said exactly controllable in time $\tau>0$ if for every $q_{0} \in \mathcal{H}_{\frac{1}{2}}, q_{1} \in \mathcal{H}$ there exists a control $u \in L^{2}([0, \tau], \mathcal{U})$ such that $q(\tau)=0$ and $\dot{q}(\tau)=0$.

On the other hand, from (2.4) it follows that if we choose the control in the feedback form

$$
u(t)=-B_{0}^{*} u(t) \quad(t \geqslant 0),
$$

where $B_{0}^{*} \in \mathcal{L}(\mathcal{H}, \mathcal{U})$ stands for the adjoint of $B_{0}$, then the energy of the system, i.e., the function

$$
t \mapsto \frac{1}{2}\left(\|\dot{w}(t)\|^{2}+\|w(t)\|_{\frac{1}{2}}^{2}\right) \quad(t \geqslant 0)
$$

is non increasing. This property of the above feedback, corresponding to collocated actuators and sensors, suggests considering the second order differential equation

$$
\begin{gathered}
\ddot{w}(t)+A_{0} w(t)+B_{0} B_{0}^{*} \dot{w}(t)=0 \quad(t \geqslant 0), \\
w(0)=w_{0}, \dot{w}(0)=w_{1} .
\end{gathered}
$$

It is well known that the above equation defines a well posed dynamical system in the state space $X=\mathcal{H}_{\frac{1}{2}} \times \mathcal{H}$. More precisely, the solution $\left[\begin{array}{c}w \\ \dot{w}\end{array}\right]$ of $(2.6),(2.7)$ is given by

$$
\left[\begin{array}{l}
w(t) \\
\dot{w}(t)
\end{array}\right]=\mathbb{T}_{t}\left[\begin{array}{l}
w_{0} \\
w_{1}
\end{array}\right] \quad\left(\left[\begin{array}{l}
w_{0} \\
w_{1}
\end{array}\right] \in X, \quad t \geqslant 0\right)
$$

where $\mathbb{T}$ is the contraction semigroup on $X$ generated by $\mathcal{A}-\mathcal{B B}^{*}$ and $\mathcal{A}: \mathcal{D}(\mathcal{A}) \rightarrow X$, $\mathcal{B} \in \mathcal{L}(\mathcal{U}, X)$ are defined by

$$
\mathcal{D}(\mathcal{A})=\mathcal{H}_{1} \times \mathcal{H}_{\frac{1}{2}}, \quad \mathcal{A}=\left[\begin{array}{cc}
0 & I \\
-A_{0} & 0
\end{array}\right], \quad \mathcal{B}=\left[\begin{array}{c}
0 \\
B_{0}
\end{array}\right] .
$$

We also consider the backwards system

$$
\begin{gathered}
\ddot{w}_{b}(t)+A_{0} w_{b}(t)-B_{0} B_{0}^{*} \dot{w}_{b}(t)=0 \quad(t \leqslant \tau), \\
w_{b}(\tau)=w(\tau), \dot{w}_{b}(\tau)=\dot{w}(\tau) .
\end{gathered}
$$

It is not difficult to check that the solution $\left[\begin{array}{l}w_{b} \\ w_{b}\end{array}\right]$ of $(2.9),(2.10)$ is given by

$$
\left[\begin{array}{l}
w_{b}(t) \\
\dot{w}_{b}(t)
\end{array}\right]=\mathbb{S}_{\tau-t}\left[\begin{array}{l}
w(\tau) \\
\dot{w}(\tau)
\end{array}\right] \quad(t \in[0, \tau])
$$


where $\mathbb{S}$ is the contraction semigroup in $X$ generated by $-\mathcal{A}-\mathcal{B B}^{*}$.

We define $L_{\tau} \in \mathcal{L}(X)$ by

$$
L_{\tau}\left[\begin{array}{l}
w_{0} \\
w_{1}
\end{array}\right]=\left[\begin{array}{l}
w_{b}(0) \\
\dot{w}_{b}(0)
\end{array}\right] \quad\left(\left[\begin{array}{l}
w_{0} \\
w_{1}
\end{array}\right] \in X\right) .
$$

With the above notation, the operator $L_{\tau}$ clearly satisfies $L_{\tau}=\mathbb{S}_{\tau} \mathbb{T}_{\tau}$.

Remark 2.1. If the semigroup $\mathbb{T}$ is exponentially stable then the same property holds for $\mathbb{S}$. Indeed, this follows from the fact that, after changing the sense of the time, the solution $w_{b}$ of the backwards problem satisfies the same initial value problem as $w$. In this case there exists $\tau>0$ such that

$$
\left\|\mathbb{T}_{\tau}\right\|_{\mathcal{L}(X)}, \quad\left\|\mathbb{S}_{\tau}\right\|_{\mathcal{L}(X)}<1
$$

and, consequently, the operator $I-L_{\tau}$ is invertible. Its inverse is given by

$$
\left(I-L_{\tau}\right)^{-1}=\sum_{n \geqslant 0} L_{\tau}^{n}
$$

A particular case of Russell's principle [29], of interest for this work, is given by the following result:

Proposition 2.2. Assume that the semigroup $\mathbb{T}$ is exponentially stable and let $\tau>0$ be such that (2.13) holds. Then the system (2.2)-(2.3) is exctly controllable in time $\tau$ and a control $u \in C([0, \tau] ; \mathcal{U})$ steering the initial state $\left[\begin{array}{c}q_{0} \\ q_{1}\end{array}\right] \in X$ to rest in time $\tau$ is given by

$$
u=B_{0}^{*} \dot{w}+B_{0}^{*} \dot{w}_{b}
$$

where $w$ and $w_{b}$ are the solutions of (2.6)-(2.7) and (2.9)-(2.10), respectively, with

$$
\left[\begin{array}{l}
w_{0} \\
w_{1}
\end{array}\right]=\left(I-L_{\tau}\right)^{-1}\left[\begin{array}{l}
q_{0} \\
q_{1}
\end{array}\right]
$$

Proof. Denote

$$
q(t)=w(t)-w_{b}(t) \quad(t \in[0, \tau]) .
$$

Then $q$ clearly satisfies (2.2) with $u$ given by (2.15). Moreover, from (2.16) it follows that $q$ satisfies the initial conditions (2.3). Finally, from (2.10) it follows that

$$
q(\tau)=\dot{q}(\tau)=0
$$

Remark 2.3. As above, the original assumption of Russell's principle was the exponential stability of the semigroup $\mathbb{T}$, whence the name "stabilizability implies controllability". The fact that, in the context of systems considered in Proposition 2.2, exponential stabilizability and exact controllability are equivalent has been remarked by direct methods in Haraux [15] and Liu [23]. The more precise fact that if the system (2.2)-(2.3) is exactly controllable in time $\tau$ then $\left\|\mathbb{T}_{\tau}\right\|_{\mathcal{L}(X)}<1$ and $\left\|\mathbb{S}_{\tau}\right\|_{\mathcal{L}(X)}<1$ is easy to establish (see, for instance, Lemma 2.2 in Ito, Ramdani and Tucsnak [16]). 
An important property of the control $u$ constructed in (2.15) is that, under appropriate assumptions on $B_{0}$, its regularity increases when the initial data are more regular. This kind of regularity property is important for approximation purposes and has been recently investigated for HUM controls. In [7] it is shown that, for the wave equation with boundary control, the HUM controls need to be modified to obtain the regularity property. In the case of the wave equation with internal control, it is shown in Lebeau and Nodet [21] and Dehman and Lebeau [5] that, under assumptions on $B_{0}$ which are similar to ours, the HUM controls are smoother if we increase the regularity of the initial data. With appropriate assumptions, the controls constructed via Russsell's principle also satisfy this regularity property, as shown by the result below:

Proposition 2.4. Assume that

$$
B_{0} B_{0}^{*} \in \mathcal{L}\left(\mathcal{H}_{k}, \mathcal{H}_{k-\frac{1}{2}}\right) \quad(k \in\{1, \ldots, m\}),
$$

and $\left[\begin{array}{l}q_{0} \\ q_{1}\end{array}\right] \in \mathcal{H}_{m+\frac{1}{2}} \times \mathcal{H}_{m}$ for some $m \in \mathbb{Z}, m \geqslant 1$. Then the controls constructed in Proposition 2.2 satisfy

$$
u \in C^{m}([0, \tau] ; \mathcal{U}), \quad B_{0} u \in C\left([0, \tau] ; \mathcal{H}_{m-\frac{1}{2}}\right) .
$$

Proof. We prove the result for $m=1$. The heneral case can be tackled by simple adaptations of the proof bellow.

First note that from (2.17) it follows that

$$
\mathcal{D}\left(\mathcal{A}-\mathcal{B B}^{*}\right)=\mathcal{H}_{1} \times \mathcal{H}_{\frac{1}{2}}, \quad \mathcal{D}\left(\left(\mathcal{A}-\mathcal{B B}^{*}\right)^{2}\right)=\mathcal{H}_{\frac{3}{2}} \times \mathcal{H}_{1}
$$

Thus, under the assumptions of Proposition 2.2, the restrictions of the semigroups $\mathbb{T}$ and $\mathbb{S}$ to $\mathcal{H}_{1} \times \mathcal{H}_{\frac{1}{2}}$ and $\mathcal{H}_{\frac{3}{2}} \times \mathcal{H}_{1}$ are contraction semigroups on these spaces. Moreover, their generators are the restrictions of $\mathcal{A}-\mathcal{B B}^{*}$ and $-\mathcal{A}-\mathcal{B B}^{*}$ to $\mathcal{H}_{\frac{3}{2}} \times \mathcal{H}_{1}$ and $\mathcal{H}_{2} \times \mathcal{H}_{\frac{3}{2}}$, respectively, and they satisfy

$$
\left\|\mathbb{T}_{\tau}\right\|_{\mathcal{L}\left(\mathcal{H}_{\frac{3}{2}} \times \mathcal{H}_{1}\right)}<1, \quad\left\|\mathbb{S}_{\tau}\right\|_{\mathcal{L}\left(\mathcal{H}_{\frac{3}{2}} \times \mathcal{H}_{1}\right)}<1 .
$$

Consequently, $I-L_{\tau} \in \mathcal{L}\left(\mathcal{H}_{\frac{3}{2}} \times \mathcal{H}_{1}\right)$ is invertible and $\left[\begin{array}{c}w_{0} \\ w_{1}\end{array}\right]:=\left(I-L_{\tau}\right)^{-1}\left[\begin{array}{l}q_{0} \\ q_{1}\end{array}\right] \in \mathcal{H}_{\frac{3}{2}} \times \mathcal{H}_{1}$. These fact imply that

$$
w, w_{b} \in C\left([0, \tau] ; \mathcal{H}_{\frac{3}{2}}\right) \cap C^{1}\left([0, \tau] ; \mathcal{H}_{1}\right),
$$

so that the control $u$ defined by (2.15) satisfies the required properties.

Remark 2.5. The example of the wave equation with distributed control, considered in [5, 21], fits in the above framework, with the below spaces and operators:

- $\mathcal{H}=L^{2}(\Omega)$, where $\Omega \subset \mathbb{R}^{n}$ is an open bounded set with smooth boundary.

- $\mathcal{U}=L^{2}(\Omega)$ and $B_{0} \in \mathcal{L}(\mathcal{U}, \mathcal{H})$ is defined by

$$
B_{0} \mathrm{v}=\mathrm{v} \chi_{\mathcal{O}} \quad(\mathrm{v} \in \mathcal{U}),
$$

where $\mathcal{O}$ is an open subset of $\Omega$ satisfying the geometric optics condition (see Bardos, Lebeau and Rauch [2]), $H^{m}(\Omega)$ are the usual Sobolev spaces, whereas $\chi_{\mathcal{O}} \in C^{\infty}(\bar{\Omega})$ is positive on $\Omega$ and strictly positive on $\mathcal{O}$. 
In this case, in particular, provided that the initial data satisfy

$$
q_{0} \in H^{3}(\Omega) \cap H_{0}^{1}(\Omega), \quad \Delta q_{0} \in H_{0}^{1}(\Omega), \quad q_{1} \in H^{2}(\Omega) \cap H_{0}^{1}(\Omega),
$$

the control constructed using Russell's principle shares the same property as the HUM controls constructed in [5, 21], which are

$$
u \in C^{1}\left([0, \tau] ; L^{2}(\Omega)\right) \cap C\left([0, \tau], H^{1}(\Omega)\right) .
$$

\section{Some background on a class of second order systems with collocated sensors and actuators}

In this section we recall some results from Tucsnak and Weiss [33, 31], Ito, Ramdani and Tucsnak [18] and Ammari and Tucsnak [1], which will play an important role in the sequel. The only (slight) novelty we bring in this section consists in providing more precise estimates on the constants involved with these results.

We continue to use the notation described in Section 2 concerning Hilbert spaces $\mathcal{H}$ and $\mathcal{U}$ and the strictly positive operator $A_{0}$ that can be defined on the scale of Hilbert spaces $\left(\mathcal{H}_{\alpha}\right)_{\alpha \in \mathbb{R}}$. A novelty with respect to the previous sections is that we allow unbounded input operators. These operators are defined by duality from an operator $C \in \mathcal{L}\left(\mathcal{H}_{\frac{1}{2}}, \mathcal{U}\right)$. More precisely, consider the system described by

$$
\begin{gathered}
\ddot{w}(t)+A_{0} w(t)+C^{*} \frac{\mathrm{d}}{\mathrm{d} t} C w(t)=C^{*} u(t), \\
y(t)=\frac{\mathrm{d}}{\mathrm{d} t} C w(t),
\end{gathered}
$$

where $t \in[0, \infty)$ is the time. The equation (3.1) is understood as an equation in $\mathcal{H}_{-\frac{1}{2}}$, i.e., all the terms are expected to be $\mathcal{H}_{-\frac{1}{2}}$. The state $z(t)$ of this system and its state space $X$ are defined by

$$
z(t)=\left[\begin{array}{c}
w(t) \\
\dot{w}(t)
\end{array}\right], \quad X=\mathcal{H}_{\frac{1}{2}} \times \mathcal{H} .
$$

We will need the following result, which can be obtained by simple algebraic manipulations from Theorem 1.1 in [33].

Theorem 3.1. For every input function $u \in L^{2}([0, \infty) ; \mathcal{U})$ and every initial state $\left[\begin{array}{l}w(0) \\ \dot{w}(0)\end{array}\right]=\left[\begin{array}{l}\psi_{0} \\ \psi_{1}\end{array}\right] \in X$ there exists a function $w$ such that

$$
w \in B C^{0}\left([0, \infty) ; \mathcal{H}_{\frac{1}{2}}\right) \cap B C^{1}([0, \infty) ; \mathcal{H}) \cap H_{l o c}^{2}\left(0, \infty ; \mathcal{H}_{-\frac{1}{2}}\right),
$$

where $B C^{n}([0, \infty) ; W)$ denotes the space of those $f \in C^{n}([0, \infty) ; W)$ for which $f, f^{\prime}, \ldots f^{(n)}$ are all bounded on $[0, \infty)$.

(2) $C w \in H^{1}((0, \infty) ; \mathcal{U})$ and the equations (3.1) and (3.2) hold for almost every $t \geqslant 0$ in $\mathcal{H}_{-\frac{1}{2}}$ and $\mathcal{U}$, respectively (hence, $y \in L^{2}([0, \infty) ; \mathcal{U})$ ).

(3) For every $t \geqslant 0$

$$
\left\|\left[\begin{array}{c}
w_{0} \\
w_{1}
\end{array}\right]\right\|_{X}^{2}-\left\|\left[\begin{array}{c}
w(t) \\
\dot{w}(t)
\end{array}\right]\right\|_{X}^{2}=2 \int_{0}^{t}\left\|\frac{\mathrm{d}}{\mathrm{d} \sigma} C w(\sigma)\right\|_{\mathcal{U}}^{2} \mathrm{~d} \sigma-2 \int_{0}^{t}\left\langle u(\sigma), \frac{\mathrm{d}}{\mathrm{d} \sigma} C w(\sigma)\right\rangle_{\mathcal{U}} \mathrm{d} \sigma .
$$


The stability properties of the system (3.1)-(3.2) can be described using the following additional assumptions on $A_{0}$ and $C$ :

(H1) There exists $\gamma>0$ such that

$$
\left\|s C\left(s^{2} I+A_{0}\right)^{-1} C^{*}\right\|_{\mathcal{L}(\mathcal{U})} \leqslant d_{\gamma} \quad(\operatorname{Re} s=\gamma)
$$

with $d_{\gamma}>0$ depending only on $\gamma$;

(H2) There exists $\tau>0, K_{\tau}>0$ such that the inequality

$$
K_{\tau}^{2} \int_{0}^{\tau}\|C \dot{p}(t)\|^{2} \mathrm{~d} t \geqslant\|f\|_{\frac{1}{2}}^{2}+\|g\|^{2}
$$

holds for every $f \in \mathcal{H}_{1}, g \in \mathcal{H}_{\frac{1}{2}}$ and $p$ satisfying

$$
\begin{gathered}
\ddot{p}(t)+A_{0} p(t)=0 \quad(t \geqslant 0), \\
p(0)=f, \quad \dot{p}(0)=g .
\end{gathered}
$$

The main result in this section is:

Theorem 3.2. Assume that $A_{0}$ and $C$ satisfy the assumption $(\mathrm{H} 1)$ and $(\mathrm{H} 2)$ above. Then there exist $m_{\tau, \gamma} \in(0,1)$, depending only on $\gamma, d_{\gamma}, \tau$ and $K_{\tau}$ such that the estimate

$$
\|\dot{w}(\tau)\|^{2}+\|w(\tau)\|_{\frac{1}{2}}^{2} \leqslant m_{\tau, \gamma}\left(\|\dot{w}(0)\|^{2}+\|w(0)\|_{\frac{1}{2}}^{2}\right)
$$

holds for every solution $w \in C\left([0, \infty) ; \mathcal{H}_{\frac{1}{2}}\right) \cap C^{1}([0, \infty) ; \mathcal{H})$ of $(3.1)$ with $u=0$.

The above theorem is essentially a consequence of Theorem 2.2 from [1], but it gives more information on the constant $m_{\tau, \gamma}$ in (3.8). Note that, using the semigroup property, this theorem is equivalent to the exponential decay of the solutions of (3.1) at a rate depending on $\tau$ and $\gamma$ only. For the sake of completeness, we give below the proof. The first step is the result below, concerning the control system

$$
\begin{gathered}
\ddot{y}(t)+A_{0} y(t)=C^{*} v(t) \quad(t \geqslant 0) \\
y(0)=0, \quad \dot{y}(0)=0 .
\end{gathered}
$$

Proposition 3.3. With the above notation, assume that the pair $\left(A_{0}, C\right)$ satisfies assumption $(\mathrm{H} 1)$. Then for every $T>0$ and $v \in L^{2}([0, T] ; \mathcal{U})$ the initial and boundary value problem (3.9)-(3.10) admits a unique solution

$$
y \in C\left([0, T] ; \mathcal{H}_{\frac{1}{2}}\right) \cap C^{1}([0, T] ; \mathcal{H}) .
$$

Moreover, the function $t \mapsto C y(t)$ is in $H^{1}((0, T) ; \mathcal{U})$ and there exists a positive constant $c_{T, \gamma}$, depending only on $T$ and on the constants $\gamma$ and $d_{\gamma}$ in (3.4), such that

$$
\left\|\frac{\mathrm{d}}{\mathrm{d} t} C y\right\|_{L^{2}([0, T] ; \mathcal{U})} \leqslant c_{T, \gamma}\|v\|_{L^{2}([0, T] ; \mathcal{U})} \quad\left(v \in L^{2}([0, T] ; \mathcal{U})\right) .
$$


Proof. The fact that (3.9)-(3.10) admits a unique solution satisfying (3.11) follows directly from Propositions 3.2 and 3.3 in [1].

To prove (3.12), we follow the procedure in [1], by carefully estimating the involved constants. To this aim we first remark that, since equation (3.9) is time reversible, after extending $v$ by zero for $t \in \mathbb{R} \backslash[0, T]$, we can solve (3.9)-(3.10) for $t \in \mathbb{R}$. We obtain in this way a function, still denoted by $y$, satisfying

$$
\begin{array}{lr}
y \in B C^{0}\left(\mathbb{R} ; \mathcal{H}_{\frac{1}{2}}\right) \cap B C^{1}(\mathbb{R} ; \mathcal{H}), \\
y(t)=0 & (t \leqslant 0),
\end{array}
$$

and $y$ satisfies (3.9) for every $t \in \mathbb{R}$. Thus the Laplace transform $\widehat{y}$ of $y$ is defined in the right half-plane and it satisfies :

$$
s^{2} \widehat{y}(s)+A_{0} \widehat{y}(s)=C^{*} \widehat{v}(s) \quad(\operatorname{Re} s>0) .
$$

The above formula and (3.4) imply that

$$
\int_{-\infty}^{\infty}\|(\gamma+i \eta) C \widehat{y}(\gamma+i \eta)\|_{\mathcal{U}}^{2} \mathrm{~d} \eta \leqslant d_{\gamma}^{2} \int_{-\infty}^{+\infty}\|\widehat{v}(\gamma+i \eta)\|_{\mathcal{U}}^{2} \mathrm{~d} \eta \quad\left(v \in L^{2}([0, T] ; \mathcal{U})\right) .
$$

Using the Parseval identity (see for instance Doetsch [6, p.212]), it follows that the map $t \mapsto e^{-\gamma t} C y(t)$ lies in $H^{1}((\mathbb{R} ; \mathcal{U}))$ and

$$
\int_{0}^{T}\left\|e^{-\gamma t} \frac{\mathrm{d}}{\mathrm{d} t}(C y(t))\right\|_{\mathcal{U}}^{2} \mathrm{~d} t \leqslant d_{\gamma}^{2} \int_{0}^{T}\|v(t)\|_{\mathcal{U}}^{2} \mathrm{~d} t \quad\left(v \in L^{2}([0, T] ; \mathcal{U})\right),
$$

which clearly implies that we have estimate (3.12).

Corollary 3.4. Under the assumptions of Proposition 3.3, let $w \in C\left([0, \infty) ; \mathcal{H}_{\frac{1}{2}}\right) \cap$ $C^{1}([0, \infty) ; \mathcal{H})$ be a solution of $(3.1)$ with $u=0$. Let $p$ be the solution of (3.6), (3.7) with $f=w(0)$ and $g=\dot{w}(0)$. Then for every $T>0$ the function $t \mapsto C p(t)$ is in $H^{1}((0, T) ; \mathcal{U})$. Moreover, there exists $M_{T, \gamma}$, depending only on $T$ and on the constants $\gamma$ and $d_{\gamma}$ in $(\mathrm{H} 1)$ such that

$$
\int_{0}^{T}\left\|\frac{\mathrm{d}}{\mathrm{d} t}(C p(t))\right\|_{\mathcal{U}}^{2} \mathrm{~d} t \leqslant M_{T, \gamma}^{2} \int_{0}^{T}\left\|\frac{\mathrm{d}}{\mathrm{d} t}(C w(t))\right\|_{\mathcal{U}}^{2} \mathrm{~d} t \quad\left(f \in \mathcal{H}_{\frac{1}{2}}, g \in \mathcal{H}\right) .
$$

Proof. For the proof of the fact that $C p \in H^{1}((0, T) ; \mathcal{U})$ we refer to Proposition 3.3 in [1].

In order to prove (3.14) we note that $w$ can be written as

$$
w(t)=p(t)+\psi(t)
$$

where $p$ satisfies (3.6), (3.7) and $\psi$ satisfies

$$
\begin{gathered}
\ddot{\psi}(t)+A_{0} \psi(t)=-C^{*} \frac{\mathrm{d}}{\mathrm{d} t} C w(t) \quad(t \in[0, T]), \\
\psi(0)=0, \quad \dot{\psi}(0)=0 .
\end{gathered}
$$

By combining (3.15) and Proposition 3.3 (with $v=\frac{\mathrm{d}}{\mathrm{d} t}(C p)$ ), it follows that

$$
\int_{0}^{T}\left\|\frac{\mathrm{d}}{\mathrm{d} t}(C p(t))\right\|_{\mathcal{U}}^{2} \mathrm{~d} t \leqslant 2\left(1+c_{T, \gamma}^{2}\right) \int_{0}^{T}\left\|\frac{\mathrm{d}}{\mathrm{d} t}(C w(t))\right\|_{\mathcal{U}}^{2} \mathrm{~d} t
$$

which ends the proof. 
We are now in a position to prove the main result in this section.

Proof of Theorem 3.2. By combining Corollary 3.4 and (3.5) it follows that

$$
\int_{0}^{\tau}\left\|\frac{\mathrm{d}}{\mathrm{d} t}(C w(t))\right\|_{\mathcal{U}}^{2} \mathrm{~d} t \geqslant M_{\tau, \gamma}^{-2} K_{\tau}^{-2}\left(\|\dot{w}(0)\|^{2}+\|w(0)\|_{\frac{1}{2}}^{2}\right),
$$

for every solution $w \in C\left([0, \infty) ; \mathcal{H}_{\frac{1}{2}}\right) \cap C^{1}([0, \infty) ; \mathcal{H})$ of $(3.1)$ with $u=0$.

On the other hand, these solutions satisfy, according to (3.3)

$$
\|\dot{w}(0)\|^{2}+\|w(0)\|_{\frac{1}{2}}^{2}-\|\dot{w}(\tau)\|^{2}-\|w(\tau)\|_{\frac{1}{2}}^{2}=2 \int_{0}^{\tau}\left\|\frac{\mathrm{d}}{\mathrm{d} \sigma}(C w(\sigma))\right\|_{\mathcal{U}}^{2} \mathrm{~d} \sigma .
$$

The above formula and (3.18) yield (3.8) with

$$
m_{\tau, \gamma}=1-2 M_{\tau, \gamma}^{-2} K_{\tau}^{-2} .
$$

\section{An abstract perturbation result}

In this section we give an abstract result of singular perturbation type for the class of systems introduced in the previous section (see Theorem 4.1 below). Our motivation is to study the behavior of a family of locally distributed control systems as the support of the control region "shrinks" to a portion of the boundary. Our main abstract result, Theorem is general enough to encompass Theorem 5.1 or Theorem 6.1 , which tackle systems governed by the string equation with Neumann or Dirichlet boundary conditions.

We continue to use below the notation introduced in Section 3, which means, in particular, that $A_{0}: \mathcal{D}\left(A_{0}\right) \rightarrow \mathcal{H}$ is strictly positive and that $B_{0} \in \mathcal{L}\left(\mathcal{U}, \mathcal{H}_{-\frac{1}{2}}\right)$ is a possibly unbounded input operator.

Let $\varepsilon_{0}>0$ and let $\left(B_{\varepsilon}\right)_{\varepsilon \in\left(0, \varepsilon_{0}\right)} \subset \mathcal{L}(\mathcal{U}, \mathcal{H})$ be a family of bounded input operators.

We consider a family of controlled systems described by the equation

$$
\begin{gathered}
\ddot{q}_{\varepsilon}(t)+A_{0} q_{\varepsilon}(t)=B_{\varepsilon} u(t) \quad\left(\varepsilon \in\left(0, \varepsilon_{0}\right), t \geqslant 0\right), \\
q_{\varepsilon}(0)=f, \quad \dot{q}_{\varepsilon}(0)=g .
\end{gathered}
$$

We will also need to refer to the corresponding uncontrolled homogeneous system

$$
\begin{gathered}
\ddot{\varphi}(t)+A_{0} \varphi(t)=0 \quad(t \geqslant 0), \\
\varphi(0)=f, \quad \dot{\varphi}(0)=g .
\end{gathered}
$$

Our main new abstract result is the following. 
Theorem 4.1. With the above notation and assumptions, suppose that there exists $\tau>0$ and $K_{\tau}>0$, depending only on $\tau$, such that the solution $\varphi$ of (4.3)-(4.4) satisfies

$$
K_{\tau}^{2} \int_{0}^{\tau}\left\|B_{\varepsilon}^{*} \dot{\varphi}(t)\right\|_{\mathcal{U}}^{2} \mathrm{~d} t \geqslant\|f\|_{\frac{1}{2}}^{2}+\|g\|_{\mathcal{H}}^{2} \quad\left(\varepsilon \in\left(0, \varepsilon_{0}\right), \quad f \in \mathcal{H}_{\frac{1}{2}}, g \in \mathcal{H}\right) .
$$

Moreover, assume that for some $\gamma>0$ there exist $\varepsilon_{0}>0$ and $d_{\gamma}>0$ such that

$$
\left\|s B_{\varepsilon}^{*}\left(s^{2} I+A_{0}\right)^{-1} B_{\varepsilon}\right\|_{\mathcal{L}(\mathcal{U})} \leqslant d_{\gamma} \quad\left(\varepsilon \in\left(0, \varepsilon_{0}\right), \text { Re } s=\gamma\right) .
$$

Finally, let $\mathcal{U}_{0}$ be another Hilbert space and assume that

$$
\lim _{\varepsilon \rightarrow 0^{+}} B_{\varepsilon} B_{\varepsilon}^{*} f=B_{0} B_{0}^{*} f \quad \text { in } \mathcal{H}_{-\frac{1}{2}} \quad\left(f \in \mathcal{H}_{\frac{1}{2}}\right),
$$

for some $B_{0} \in \mathcal{L}\left(\mathcal{U}_{0}, \mathcal{H}_{-\frac{1}{2}}\right)$. Then for every $f \in \mathcal{H}_{\frac{1}{2}}$ and $g \in \mathcal{H}$ there exists a family of controls $\left(u_{\varepsilon}\right)_{\varepsilon \in\left(0, \varepsilon_{0}\right)}$ in $L^{2}([0, \tau] ; \mathcal{U})$ such that

1. The corresponding family $\left(q_{\varepsilon}\right)$ of solutions of (4.1)-(4.2) satisfies

$$
q_{\varepsilon}(\tau)=0, \quad \dot{q}_{\varepsilon}(\tau)=0 \quad\left(\varepsilon \in\left(0, \varepsilon_{0}\right)\right) .
$$

2. There exists $u_{0} \in L^{2}\left([0, \tau] ; \mathcal{U}_{0}\right)$ such that the solution of

$$
\ddot{q}_{0}(t)+A_{0} q_{0}(t)=B_{0} u_{0}, \quad q_{0}(0)=f, \dot{q}_{0}(0)=g
$$

satisfies $q_{0}(\tau)=0, \dot{q}_{0}(\tau)=0$ and

$$
\lim _{\varepsilon \rightarrow 0^{+}} B_{\varepsilon} u_{\varepsilon}=B_{0} u_{0} \quad \text { weakly in } \quad L^{2}\left([0, \tau] ; \mathcal{H}_{-\frac{1}{2}}\right) .
$$

3. The corresponding controlled trajectories satisfy

$$
\lim _{\varepsilon \rightarrow 0^{+}}\left(\left\|q_{\varepsilon}-q_{0}\right\|_{C\left([0, \tau] ; \mathcal{H}_{\frac{1}{2}}\right)}+\left\|\dot{q}_{\varepsilon}-\dot{q}_{0}\right\|_{C([0, \tau] ; \mathcal{H})}\right)=0 .
$$

We remark that the hypotheses (4.5) and (4.6) of Theorem 4.1 describe respectively, uniform observability with respect to $\varepsilon \in\left(0, \varepsilon_{0}\right)$ and uniform well-posedness in the sense of Weiss with respect to $\varepsilon \in\left(0, \varepsilon_{0}\right)$, for the system (4.1), ( 4.2) with state space $X=\mathcal{H}_{\frac{1}{2}} \times \mathcal{H}$, input and output space $\mathcal{U}$ and output function $y(t)=B_{\varepsilon}^{*} \dot{\varphi}(t)$.

We consider the family of initial value problems

$$
\begin{array}{cl}
\ddot{w}_{\varepsilon}(t)+A_{0} w_{\varepsilon}(t)+B_{\varepsilon} \frac{\mathrm{d}}{\mathrm{d} t}\left(B_{\varepsilon}^{*} w_{\varepsilon}(t)\right)=0 & \left(\varepsilon \in\left[0, \varepsilon_{0}\right), t \geqslant 0\right), \\
w_{\varepsilon}(0)=\psi_{0}, \quad \dot{w}_{\varepsilon}(0)=\psi_{1} \quad\left(\varepsilon \in\left[0, \varepsilon_{0}\right)\right) .
\end{array}
$$

By applying Theorem 3.1, with $C=B_{\varepsilon}^{*}$, it follows that for every $\varepsilon \in\left[0, \varepsilon_{0}\right), \psi_{0} \in \mathcal{H}_{\frac{1}{2}}$ and $\psi_{1} \in \mathcal{H}$ the system (4.11)-(4.12) admits a unique solution

$$
w_{\varepsilon} \in B C^{0}\left([0, \infty) ; \mathcal{H}_{\frac{1}{2}}\right) \cap B C^{1}([0, \infty) ; \mathcal{H}) \cap H_{l o c}^{2}\left(0, \infty ; \mathcal{H}_{-\frac{1}{2}}\right),
$$

with $B_{\varepsilon}^{*} w_{\varepsilon} \in H_{\mathrm{loc}}^{1}((0, \infty) ; \mathcal{U})$ and

$$
2 \int_{0}^{\infty}\left\|\frac{\mathrm{d}}{\mathrm{d} \sigma}\left(B_{\varepsilon}^{*} w_{\varepsilon}(\sigma)\right)\right\|_{\mathcal{U}}^{2} \mathrm{~d} \sigma \leqslant\left\|\psi_{0}\right\|_{\frac{1}{2}}^{2}+\left\|\psi_{1}\right\|^{2} \quad\left(\psi_{0} \in \mathcal{H}_{\frac{1}{2}}, \quad \psi_{1} \in \mathcal{H}\right) .
$$


Proposition 4.2. With the above notation, assume that the operators $\left(B_{\varepsilon}\right)_{\varepsilon \geqslant 0}$ satisfy (4.7). Then for every $\tau>0, \psi_{0} \in \mathcal{H}_{\frac{1}{2}}$ and $\psi_{1} \in \mathcal{H}$, the solutions $\left(w_{\varepsilon}\right)_{\varepsilon \in\left[0, \varepsilon_{0}\right)}$ of (4.11), (4.12) satisfy

$$
\begin{gathered}
\lim _{\varepsilon \rightarrow 0^{+}}\left(\left\|w_{\varepsilon}-w_{0}\right\|_{C\left([0, \tau] ; \mathcal{H}_{\frac{1}{2}}\right)}+\left\|\dot{w}_{\varepsilon}-\dot{w}_{0}\right\|_{C([0, \tau] ; \mathcal{H})}\right)=0, \\
\lim _{\varepsilon \rightarrow 0^{+}}\left[B_{\varepsilon} \frac{\mathrm{d}}{\mathrm{d} t}\left(B_{\varepsilon}^{*} w_{\varepsilon}\right)\right]=\left[B_{0} \frac{\mathrm{d}}{\mathrm{d} t}\left(B_{0}^{*} w_{0}\right)\right] \quad \text { weakly in } L^{2}\left([0, \tau] ; \mathcal{H}_{-\frac{1}{2}}\right) .
\end{gathered}
$$

Proof. In order to prove (4.14) we write (4.11), (4.12) as a first order system in $X=\mathcal{H}_{\frac{1}{2}} \times \mathcal{H}$ by introducing, for every $\varepsilon \in\left[0, \varepsilon_{0}\right)$, the operator $\mathcal{A}_{\varepsilon}: \mathcal{D}\left(\mathcal{A}_{\varepsilon}\right) \rightarrow X$ defined by

$$
\begin{gathered}
\mathcal{A}_{\varepsilon}=\left(\begin{array}{cc}
0 & I \\
-A_{0} & -B_{\varepsilon} B_{\varepsilon}^{*}
\end{array}\right), \\
\mathcal{D}\left(\mathcal{A}_{\varepsilon}\right)=\left\{\left[\begin{array}{l}
f \\
g
\end{array}\right] \in \mathcal{H}_{\frac{1}{2}} \times \mathcal{H}_{\frac{1}{2}} \mid A_{0} f+B_{\varepsilon} B_{\varepsilon}^{*} g \in \mathcal{H}\right\} .
\end{gathered}
$$

It is well known that $\mathcal{A}_{\varepsilon}$ generates, for every $\varepsilon \in\left[0, \varepsilon_{0}\right)$, a strongly continuous contraction semigroup $\mathbb{T}^{\varepsilon}$ on $X$. This fact is obvious when $\varepsilon \in\left(0, \varepsilon_{0}\right)$ (in this case $\mathcal{A}_{\varepsilon}$ is a bounded perturbation of a skew-adjoint operator and $\left.\mathcal{D}\left(\mathcal{A}_{\varepsilon}\right)=\mathcal{H}_{1} \times \mathcal{H}_{\frac{1}{2}}\right)$ and we refer, for instance, to [33] for the case $\varepsilon=0$. Since equations (4.11), (4.12) can be written for every $\varepsilon \in\left[0, \varepsilon_{0}\right)$ in the first order form

$$
\frac{\mathrm{d}}{\mathrm{d} t}\left[\begin{array}{c}
w_{\varepsilon}(t) \\
\dot{w}_{\varepsilon}(t)
\end{array}\right]=\mathcal{A}_{\varepsilon}\left[\begin{array}{c}
w_{\varepsilon}(t) \\
\dot{w}_{\varepsilon}(t)
\end{array}\right], \quad\left[\begin{array}{c}
w_{\varepsilon}(0) \\
\dot{w}_{\varepsilon}(0)
\end{array}\right]=\left[\begin{array}{c}
\psi_{0} \\
\dot{\psi}_{1}
\end{array}\right]
$$

it follows that

$$
\left[\begin{array}{l}
w_{\varepsilon}(t) \\
\dot{w}_{\varepsilon}(t)
\end{array}\right]=\mathbb{T}_{t}^{\varepsilon}\left[\begin{array}{l}
\psi_{0} \\
\psi_{1}
\end{array}\right] \quad\left(\varepsilon \in\left(0, \varepsilon_{0}\right) t \geqslant 0\right)
$$

On the other hand, it is easy to check that for every $\varepsilon \in\left(0, \varepsilon_{0}\right)$ we have that $\mathcal{A}_{\varepsilon}$ is invertible and

$$
\mathcal{A}_{\varepsilon}^{-1}\left[\begin{array}{l}
f \\
g
\end{array}\right]=\left[\begin{array}{c}
g \\
-A_{0}^{-1}\left(f+B_{\varepsilon} B_{\varepsilon}^{*} g\right)
\end{array}\right] \quad\left(f \in \mathcal{H}_{\frac{1}{2}}, g \in \mathcal{H}\right) .
$$

The above formula, combined with (4.7) and with the fact that $A_{0}$ maps continuously $\mathcal{H}_{-\frac{1}{2}}$ onto $\mathcal{H}_{\frac{1}{2}}$, implies that

$$
\lim _{\varepsilon \rightarrow 0^{+}} \mathcal{A}_{\varepsilon}^{-1}\left[\begin{array}{l}
f \\
g
\end{array}\right]=\mathcal{A}_{0}^{-1}\left[\begin{array}{l}
f \\
g
\end{array}\right] \quad \text { in } \mathcal{H}_{\frac{1}{2}} \times \mathcal{H} \quad\left(f \in \mathcal{H}_{\frac{1}{2}}, \quad g \in \mathcal{H}\right) .
$$

By applying the Trotter-Kato theorem (see, for instance, [25, p.86]) it follows that

$$
\lim _{\varepsilon \rightarrow 0^{+}} \mathbb{T}_{t}^{\varepsilon}\left[\begin{array}{l}
\psi_{0} \\
\psi_{1}
\end{array}\right]=\mathbb{T}_{t}^{0}\left[\begin{array}{l}
\psi_{0} \\
\psi_{1}
\end{array}\right] \quad\left(\psi_{0} \in \mathcal{H}_{\frac{1}{2}}, \psi_{1} \in \mathcal{H}\right)
$$

uniformly with respect to $t$ on compact intervals, so that, using (4.18), we obtain (4.14). 
In order to prove (4.15) we note that integrating (4.11) and using (4.12) it follows that

$$
\dot{w}_{\varepsilon}(t)-\psi_{1}+A_{0} \int_{0}^{t} w_{\varepsilon}(\sigma) \mathrm{d} \sigma+B_{\varepsilon} B_{\varepsilon}^{*} w_{\varepsilon}(t)=B_{\varepsilon} B_{\varepsilon}^{*} \psi_{0} \quad\left(\varepsilon \in\left(0, \varepsilon_{0}\right), t \geqslant 0\right) .
$$

The above formula, (4.14) and the fact that $A_{0}$ maps continuously $\mathcal{H}_{\frac{1}{2}}$ on $\mathcal{H}_{-\frac{1}{2}}$ imply that

$$
\lim _{\varepsilon \rightarrow 0^{+}} B_{\varepsilon} B_{\varepsilon}^{*} w_{\varepsilon}=B_{0} B_{0}^{*} w_{0} \quad \text { in } C\left([0, \tau] ; \mathcal{H}_{-\frac{1}{2}}\right) .
$$

On the other hand, combining (4.13) with (4.7) it follows that there exists a positive constant $M_{1}$ such that

$$
\left\|B_{\varepsilon} \frac{\mathrm{d}}{\mathrm{d} t}\left(B_{\varepsilon}^{*} w_{\varepsilon}\right)\right\|_{L^{2}\left([0, \tau] ; \mathcal{H}_{-\frac{1}{2}}\right)} \leqslant M_{1} \quad\left(\varepsilon \in\left(0, \varepsilon_{0}\right)\right) .
$$

The last estimate and (4.19) imply (4.15).

We are now in a position to give the main proof of this section.

Proof of Theorem 4.1. We apply a parameter dependent (and adapted to unbounded input operators) version of Russell's "stabilizability implies controllability" principle. More precisely, for $\varepsilon \in\left[0, \varepsilon_{0}\right)$ let $\psi_{0, \varepsilon} \in \mathcal{H}_{\frac{1}{2}}, \psi_{1, \varepsilon} \in \mathcal{H}$ to be chosen later on and let $w_{\varepsilon}$, respectively $w_{\varepsilon}^{b}$, be the solution on $[0, \tau]$ of the initial, respectively the final, value problem

$$
\ddot{w}_{\varepsilon}(t)+A_{0} w_{\varepsilon}(t)+B_{\varepsilon} \frac{\mathrm{d}}{\mathrm{d} t}\left(B_{\varepsilon}^{*} w_{\varepsilon}(t)\right)=0, \quad w_{\varepsilon}(0)=\psi_{0, \varepsilon}, \quad \dot{w}_{\varepsilon}(0)=\psi_{1, \varepsilon},
$$

respectively of

$$
\ddot{w}_{\varepsilon}^{b}(t)+A_{0} w_{\varepsilon}^{b}(t)-B_{\varepsilon} \frac{\mathrm{d}}{\mathrm{d} t}\left(B_{\varepsilon}^{*} w_{\varepsilon}^{b}(t)\right)=0, \quad w_{\varepsilon}^{b}(\tau)=w_{\varepsilon}(\tau), \quad \dot{w}_{\varepsilon}^{b}(\tau)=\dot{w}_{\varepsilon}(\tau) .
$$

For $\varepsilon \in\left[0, \varepsilon_{0}\right)$ we define $L_{\tau}^{\varepsilon} \in \mathcal{L}(X)$ (recall that $X=\mathcal{H}_{\frac{1}{2}} \times \mathcal{H}$ ) by

$$
L_{\tau}^{\varepsilon}\left[\begin{array}{c}
\psi_{0, \varepsilon} \\
\psi_{1, \varepsilon}
\end{array}\right]=\left[\begin{array}{c}
w_{\varepsilon}^{b}(0) \\
\dot{w}_{\varepsilon}^{b}(0)
\end{array}\right] \quad\left(\left[\begin{array}{c}
\psi_{0, \varepsilon} \\
\psi_{1, \varepsilon}
\end{array}\right] \in X\right)
$$

According to Theorem 3.2 there exists $m_{\tau, \gamma} \in(0,1)$, depending only on $\gamma, d_{\gamma}, \tau$ and $K_{\tau}$

$$
\left\|L_{\tau}^{\varepsilon}\right\|_{\mathcal{L}(X)} \leqslant m_{\tau, \gamma} \quad\left(\varepsilon \in\left(0, \varepsilon_{0}\right)\right) .
$$

The above estimate implies that $I-L_{\tau}^{\varepsilon}$ is invertible and that

$$
\left\|\left(I-L_{\tau}^{\varepsilon}\right)^{-1}\right\|_{\mathcal{L}(X)} \leqslant \frac{1}{1-m_{\tau, \gamma}} \quad\left(\varepsilon \in\left(0, \varepsilon_{0}\right)\right) .
$$

Given $\left[\begin{array}{l}f \\ g\end{array}\right] \in X$ we choose now

$$
\left[\begin{array}{l}
\psi_{0, \varepsilon} \\
\psi_{1, \varepsilon}
\end{array}\right]=\left(I-L_{\tau}^{\varepsilon}\right)^{-1}\left[\begin{array}{l}
f \\
g
\end{array}\right] \quad\left(\varepsilon \in\left(0, \varepsilon_{0}\right)\right)
$$


This choice is motivated by the fact that if, with the above choice of $\psi_{0, \varepsilon}$ and $\psi_{1, \varepsilon}$, we set

$$
q_{\varepsilon}(t)=w_{\varepsilon}(t)-w_{b}(t) \quad\left(\varepsilon \in\left(0, \varepsilon_{0}\right), t \in[0, \tau]\right)
$$

then for every $\varepsilon \in\left(0, \varepsilon_{0}\right)$ we have

$$
\ddot{q}_{\varepsilon}(t)+A_{0} q_{\varepsilon}(t)+B_{\varepsilon} \frac{\mathrm{d}}{\mathrm{d} t}\left(B_{\varepsilon}^{*} w_{\varepsilon}(t)+B_{\varepsilon}^{*} w_{\varepsilon}^{b}(t)\right)=0, \quad\left[\begin{array}{l}
q_{\varepsilon}(0) \\
\dot{q}_{\varepsilon}(0)
\end{array}\right]=\left[\begin{array}{l}
f \\
g
\end{array}\right] \quad\left[\begin{array}{l}
q_{\varepsilon}(\tau) \\
\dot{q}_{\varepsilon}(\tau)
\end{array}\right]=\left[\begin{array}{l}
0 \\
0
\end{array}\right] .
$$

Thus for each $\varepsilon \in\left(0, \varepsilon_{0}\right)$ the control function $u_{\varepsilon} \in C([0, \tau] ; \mathcal{U})$ defined by

$$
u_{\varepsilon}(t)=-\frac{\mathrm{d}}{\mathrm{d} t}\left(B_{\varepsilon}^{*} w_{\varepsilon}(t)+B_{\varepsilon}^{*} w_{\varepsilon}^{b}(t)\right)
$$

is a control driving the initial data $\left[\begin{array}{l}f \\ g\end{array}\right]$ to rest in time $\tau$.

Let us investigate the behavior when $\varepsilon \rightarrow 0^{+}$of the solution $w_{\varepsilon}$ of $(4.20)$, with the initial data $\psi_{0, \varepsilon}, \psi_{1, \varepsilon}$ satisfying (4.24). To this aim we first note that from Proposition 4.2 it follows that $\left\|L_{\tau}^{0}\right\|_{\mathcal{L}(X)}<1$, so that $I-L_{\tau}^{0}$ is invertible. Thus, applying (4.24) and Proposition 4.2 it follows easily that

$$
\lim _{\varepsilon \rightarrow 0^{+}}\left[\begin{array}{l}
\psi_{0, \varepsilon} \\
\psi_{1, \varepsilon}
\end{array}\right]=\left(I-L_{\tau}^{0}\right)^{-1}\left[\begin{array}{l}
f \\
g
\end{array}\right] .
$$

By combining the above estimate and Proposition 4.2 it follows that

$$
\begin{gathered}
\lim _{\varepsilon \rightarrow 0^{+}}\left(\left\|w_{\varepsilon}-w_{0}\right\|_{C\left([0, \tau] ; \mathcal{H}_{\frac{1}{2}}\right)}+\left\|\dot{w}_{\varepsilon}-\dot{w}_{0}\right\|_{C([0, \tau] ; \mathcal{H})}\right)=0, \\
\lim _{\varepsilon \rightarrow 0^{+}}\left(\left\|w_{\varepsilon}^{b}-w_{0}^{b}\right\|_{C\left([0, \tau] ; \mathcal{H}_{\frac{1}{2}}\right)}+\left\|\dot{w}_{\varepsilon}^{b}-\dot{w}_{0}^{b}\right\|_{C([0, \tau] ; \mathcal{H})}\right)=0 . \\
\lim _{\varepsilon \rightarrow 0^{+}} B_{\varepsilon} \frac{\mathrm{d}}{\mathrm{d} t}\left(B_{\varepsilon}^{*} w_{\varepsilon}\right)=B_{0} \frac{\mathrm{d}}{\mathrm{d} t} B_{0}^{*} w_{0}, \quad \lim _{\varepsilon \rightarrow 0^{+}} B_{\varepsilon} \frac{\mathrm{d}}{\mathrm{d} t}\left(B_{\varepsilon}^{*} w_{\varepsilon}^{b}\right)=B_{0} \frac{\mathrm{d}}{\mathrm{d} t} B_{0}^{*} w_{0}^{b},
\end{gathered}
$$

the last convergences holding weakly in $L^{2}\left([0, \tau] ; \mathcal{H}_{-\frac{1}{2}}\right)$. By combining the last three estimates and (4.26) it follows that the family of state trajectories $\left(q_{\varepsilon}\right)$ defined in $(4.25)$, together with the family of input functions $\left(u_{\varepsilon}\right)$ defined in (4.27) satisfy the conditions required in the conclusion of our theorem.

\section{Neumann control of the string equation}

In this section we show how the system described by the string equation with Neumann boundary controlled can be approximated by a family of systems with distributed control. More precisely, for every $\varepsilon \in(0, \pi)$ we consider the control system

$$
\begin{array}{rr}
\ddot{q}_{\varepsilon}(x, t)+\frac{\partial^{2} q_{\varepsilon}}{\partial x^{2}}(x, t)+\frac{1}{\sqrt{\varepsilon}} \mathbb{1}_{[0, \varepsilon]}(x) u_{\varepsilon}(x, t)=0, \quad((x, t) \in(0, \pi) \times[0, \tau]) \\
\frac{\partial q_{\varepsilon}}{\partial x}(0, t)=q_{\varepsilon}(\pi, t)=0 \\
q_{\varepsilon}(x, 0)=f(x), \quad \dot{q}_{\varepsilon}(x, 0)=g(x) \quad(t \geqslant 0)
\end{array}
$$


where $\mathbb{1}_{[0, \varepsilon]}$ stands for the indicator function of the interval $[0, \varepsilon]$. It is known that the above system is, for every $\varepsilon>0$ exactly controllable in any time $\tau \geqslant 2 \pi$. A natural question is whether is possible to choose a sequence of controls $\left(u_{\varepsilon}\right)$ converging, in some sense to an exact control $u_{0}$ for the system

$$
\begin{array}{r}
\ddot{q}_{0}(x, t)+\frac{\partial^{2} q_{0}}{\partial x^{2}}(x, t)=0, \quad(x, t) \in \Omega \times[0, \tau] \\
\frac{\partial q_{0}}{\partial x}(0, t)=u_{0}(t), \quad \frac{\partial q_{0}}{\partial x}(\pi, t)=0, \quad t \geqslant 0 \\
q_{0}(x, 0)=f(x), \quad \dot{q}_{0}(x, 0)=g(x), \quad x \in[0, \pi] .
\end{array}
$$

Our result below provides a positive answer to the above question.

Theorem 5.1. Given $\tau \geqslant 2 \pi, f \in H^{1}(0, \pi), g \in L^{2}[0, \pi]$ with $f(\pi)=0$, there exists a family $\left(u_{\varepsilon}\right)_{\varepsilon \in(0, \pi)}$ in $L^{2}\left([0, \tau] ; L^{2}[0, \pi]\right)$ and $u_{0} \in L^{2}[0, \tau]$ such that

1. For each $\varepsilon \in(0, \pi)$ the solution of (5.1)-(5.3) satisfies

$$
q_{\varepsilon}(x, \tau)=0, \quad \dot{q}_{\varepsilon}(x, \tau)=0, \quad x \in[0, \pi]
$$

2. $\lim _{\varepsilon \rightarrow 0^{+}} \frac{1}{\sqrt{\varepsilon}} u_{\varepsilon} \mathbb{1}_{[0, \varepsilon]}=u_{0} \delta_{0}$ weakly in $L^{2}\left([0, \tau] ; H^{-1}(\mathbb{R})\right)$, where $\delta_{0}$ stands for the Dirac mass concentrated at the origin;

3. $\lim _{\varepsilon \rightarrow 0^{+}}\left(\left\|q_{\varepsilon}-q_{0}\right\|_{C\left([0, \tau] ; H^{1}(0, \pi)\right)}+\left\|\dot{q}_{\varepsilon}-\dot{q}_{0}\right\|_{C\left([0, \tau] ; L^{2}[0, \pi]\right)}\right)=0$, where $q_{0}$ is the solution of (5.4)-(5.6).

As far as we know, the approximation of Neumann boundary controls by internal controls (as in the above theorem) has not been tackled in the context of exact controllability. Joly in [19] studied this type of problem in the context of stabilization and Fabre [11] discusses the related problem of approximating pointwise internal controls at so-called "strategic points" by distributed controls for the string equation (with homogeneous Dirichlet boundary conditions). In comparison with the results in [11] where only the weak star convergence of $q_{\varepsilon}$ in $L^{\infty}\left([0, \tau] ; H^{1}(0, \pi)\right)$ is obtained, we obtain strong convergence in conclusion 3 of Theorem 5.1.

Denote $\mathcal{H}=L^{2}[0, \pi]$ and let $A_{0}$ be defined by

$$
\begin{array}{c|l}
\mathcal{D}\left(A_{0}\right)=\left\{f \in H^{2}(0, \pi)\right. & \left.\frac{\mathrm{d} f}{\mathrm{~d} x}(0)=f(\pi)=0\right\}, \\
A_{0} z=-\frac{\mathrm{d}^{2} f}{\mathrm{~d} x^{2}} \quad \forall f \in \mathcal{D}\left(A_{0}\right) .
\end{array}
$$

We denote by $\mathcal{H}_{1}$ and $\mathcal{H}_{\frac{1}{2}}$ the spaces $\mathcal{D}\left(A_{0}\right)$ and $\mathcal{D}\left(A_{0}^{\frac{1}{2}}\right)$, respectively, both endowed with the graph norm. It is known (see, for instance [32, Example 3.4.12]) that, with the above choice of spaces and operators we have

$$
\mathcal{H}_{\frac{1}{2}}=\left\{f \in H^{1}(0, \pi) \mid f(\pi)=0\right\} .
$$

Let $\mathcal{U}=L^{2}[0, \pi]$ and $\left(B_{\varepsilon}\right)_{\varepsilon>0} \subset \mathcal{L}(\mathcal{U}, \mathcal{H})$ be defined by

$$
B_{\varepsilon} u=\frac{1}{\sqrt{\varepsilon}} u \mathbb{1}_{[0, \varepsilon]} \quad(0<\varepsilon<\pi, \quad u \in \mathcal{U}) .
$$


With the above notation, the control system (5.1)-(5.3) can be rewritten as

$$
\ddot{q}_{\varepsilon}(t)+A_{0} q_{\varepsilon}(t)=B_{\varepsilon} u_{\varepsilon}(t), \quad\left[\begin{array}{c}
q_{\varepsilon}(0) \\
\dot{q}_{\varepsilon}(0)
\end{array}\right]=\left[\begin{array}{l}
f \\
g
\end{array}\right] .
$$

Next we verify that the hypothesis (4.5), (4.6), and (4.7) of Theorem 4.1 is satisfied for the system (5.1)-(5.3). We begin with verifying the assumption (4.7).

Proposition 5.2. We have

$$
\lim _{\varepsilon \rightarrow 0^{+}} B_{\varepsilon} B_{\varepsilon}^{*} \varphi=B_{0} B_{0}^{*} \varphi \quad \text { in } \mathcal{H}_{-\frac{1}{2}} \quad\left(\varphi \in \mathcal{H}_{\frac{1}{2}}\right)
$$

where $\mathcal{U}_{0}=\mathbb{C}$ and $B_{0} \in \mathcal{L}\left(\mathcal{U}_{0}, \mathcal{H}_{-\frac{1}{2}}\right)$,

$$
B_{0} u=u \delta_{0} \quad\left(u \in \mathcal{U}_{0}\right)
$$

where $\delta_{0}$ is the Dirac mass concentrated at $x=0$.

Proof. From the definition of $B_{\varepsilon}$ it follows that

$$
\left\langle B_{\varepsilon} B_{\varepsilon}^{*} \varphi, \psi\right\rangle=\frac{1}{\varepsilon} \int_{0}^{\varepsilon} \varphi(x) \psi(x) \mathrm{d} x \quad\left(\varphi, \psi \in \mathcal{H}_{\frac{1}{2}}\right) .
$$

Since

$$
\left|\frac{\psi(x)-\psi(0)}{x}\right|=\left|\frac{\int_{0}^{x} \psi^{\prime}(\xi) \mathrm{d} \xi}{x}\right| \leqslant \frac{1}{\sqrt{x}}\|\psi\|_{H^{1}(0, \pi)},
$$

it follows that $\psi(x)=\psi(0)+x \tilde{\psi}(x)$ for some $\tilde{\psi} \in L^{1}[0, \pi]$, with

$$
\|\tilde{\psi}\|_{L^{1}[0, \pi]} \leqslant K\|\psi\|_{H^{1}(0, \pi)}
$$

for some universal constant $K>0$. Consequently,

$$
\left\langle B_{\varepsilon} B_{\varepsilon}^{*} \varphi, \psi\right\rangle=\frac{1}{\varepsilon} \psi(0) \int_{0}^{\varepsilon} \varphi(x) \mathrm{d} x+\frac{1}{\varepsilon} \int_{0}^{\varepsilon} x \varphi(x) \tilde{\psi}(x) \mathrm{d} x \quad\left(\varphi, \psi \in \mathcal{H}_{\frac{1}{2}}\right) .
$$

The above formula implies that for every $\varepsilon \in(0, \pi)$ we have

$$
\begin{aligned}
\mid\left\langle B_{\varepsilon} B_{\varepsilon}^{*} \varphi, \psi\right\rangle & -\left\langle B_{0} B_{0}^{*} \varphi, \psi\right\rangle_{-\frac{1}{2}, \frac{1}{2}}\left|=\frac{1}{\varepsilon}\right| \int_{0}^{\varepsilon} x \varphi(x) \tilde{\psi}(x) \mathrm{d} x+\psi(0) \int_{0}^{\varepsilon}(\varphi(x)-\varphi(0)) \mathrm{d} x \mid \\
& \leqslant \varepsilon\|\varphi\|_{L^{\infty}[0, \pi]}\|\tilde{\psi}\|_{L^{1}[0, \pi]}+\|\psi\|_{L^{\infty}[0, \pi]}\left|\varphi\left(x_{\varepsilon}\right)-\varphi(0)\right| \quad\left(\varphi, \psi \in \mathcal{H}_{\frac{1}{2}}\right),
\end{aligned}
$$

where $0 \leqslant x_{\varepsilon} \leqslant \varepsilon$ and $B_{0}$ has been defined in (5.12). The last formula, combined with (5.13) and with the continuity of the embedding $H^{1}(0, \pi) \subset C[0, \pi]$ implies the conclusion (5.11).

Next we verify that the uniform observability condition (4.5) holds for the the system (5.1)-(5.3).

Proposition 5.3. Let $A_{0}$ and $B_{\varepsilon}$ be defined in (5.7), (5.8) and (5.9), respectively. Then for every $\tau \geqslant 2 \pi$ there exists $K_{\tau}>0$ such that the solution $\varphi$ of (4.3)-(4.4) satisfies (4.5), uniformly with respect to $\varepsilon \in(0, \pi)$. 
Proof. For $n \in \mathbb{N}$, denote $\phi_{n}(x)=\sqrt{\frac{2}{\pi}} \cos \left[\left(n-\frac{1}{2}\right) x\right]$ and $\mu_{n}=n-\frac{1}{2}$. We know that $\left(\phi_{n}\right)_{n \geqslant 1}$ is an orthonormal basis in $L^{2}[0, \pi]$ formed of eigenvectors of $A_{0}$, with corresponding eigenvalues $\left(\mu_{n}^{2}\right)_{n \geqslant 1}$. It can be easily checked that the solution of (4.3)-(4.4) can be written

$$
\varphi(x, t)=\sum_{n \geqslant 1}\left(\left\langle f, \phi_{n}\right\rangle \cos \left(\mu_{n} t\right)+\frac{1}{\mu_{n}}\left\langle g, \phi_{n}\right\rangle \sin \left(\mu_{n} t\right)\right) \phi_{n}(x) \quad(x \in(0, \pi), t \geqslant 0) .
$$

Using the definition of $B_{\varepsilon}$ it follows that for every $x \in(0, \pi)$ and $t \geqslant 0$ we have

$$
\left(B_{\varepsilon}^{*} \dot{\varphi}\right)(x, t)=\sum_{n \geqslant 1}\left(-\mu_{n}\left\langle f, \phi_{n}\right\rangle \sin \left(\mu_{n} t\right)+\left\langle g, \phi_{n}\right\rangle \cos \left(\mu_{n} t\right)\right) \phi_{n}(x) \mathbb{1}_{[0, \varepsilon]}(x) .
$$

Thus, using the fact that the family

$$
\mathcal{F}=\left(\cup_{n \geqslant 1}\left\{\sin \left(\mu_{n} t\right)\right\}\right) \cup\left(\cup_{n \geqslant 1}\left\{\cos \left(\mu_{n} t\right)\right\}\right),
$$

is orthogonal in $L^{2}[0,2 \pi]$ we deduce that for every $\tau \geqslant 2 \pi$ there exists $c_{\tau}>0$ such that

$$
\begin{aligned}
& \int_{0}^{\tau}\left\|\left(B_{\varepsilon}^{*} \dot{\varphi}\right)(\cdot, t)\right\|_{\mathcal{U}}^{2} \mathrm{~d} t \\
&=\frac{1}{\varepsilon} \int_{0}^{\varepsilon} \int_{0}^{\tau} \sum_{n \geqslant 1}\left|\left(-\mu_{n}\left\langle f, \phi_{n}\right\rangle \sin \left(\mu_{n} t\right)+\left\langle g, \phi_{n}\right\rangle \cos \left(\mu_{n} t\right)\right) \phi_{n}(x)\right|^{2} \mathrm{~d} x \mathrm{~d} t \\
& \quad \geqslant \frac{c_{\tau}}{\varepsilon} \sum_{n \geqslant 1}\left(\mu_{n}^{2}\left|\left\langle f, \phi_{n}\right\rangle\right|^{2}+\left|\left\langle g, \phi_{n}\right\rangle\right|^{2}\right) \int_{0}^{\varepsilon} \phi_{n}^{2}(x) \mathrm{d} x .
\end{aligned}
$$

Moreover, we note that

$$
\int_{0}^{\varepsilon} \phi_{n}^{2}(x) \mathrm{d} x \geqslant \frac{\varepsilon}{3} \quad(\varepsilon \in(0, \pi), n \in \mathbb{N}) .
$$

Indeed, this follows from the identity

$$
\int_{0}^{\varepsilon} \cos ^{2}(\beta x) \mathrm{d} x=\frac{\varepsilon}{2}\left(1-\frac{\sin (2 \beta \varepsilon)}{2 \beta \varepsilon}\right) \quad(\varepsilon \neq 0),
$$

together with the easy to prove inequality $\sin r>-r / 3$, which holds for every $r>0$. By combining (5.15) and (5.16) we obtain that there exists $c_{\tau}>0$ such that

$$
\int_{0}^{\tau}\left\|\left(B_{\varepsilon}^{*} \dot{\varphi}\right)(\cdot, t)\right\|_{\mathcal{U}}^{2} \mathrm{~d} t \geqslant c_{\tau}\left(\|f\|_{\frac{1}{2}}^{2}+\|g\|^{2}\right) \quad\left(\varepsilon \in\left(0, \varepsilon_{0}\right), f \in \mathcal{H}_{\frac{1}{2}}, g \in \mathcal{H}\right)
$$

which is the desired conclusion.

We are now in a position to give the main proof in this section

Proof of Theorem 5.1. We have seen in Propositions 5.2 and 5.3 that $A_{0}$ and $\left(B_{\varepsilon}\right)$ satisfy the assumptions (4.7) and (4.5) in Theorem 4.1, respectively. Accordingly, our conclusion follows from Theorem 4.1, provided that we check that $A_{0}$ and $\left(B_{\varepsilon}\right)$ also satisfy assumption (4.6) in the above mentioned theorem, i.e., if we show that for every $\alpha>0$ there exists $M_{\alpha}>0$ such that

$$
\left\|s B_{\varepsilon}^{*}\left(s^{2} I+A_{0}\right)^{-1} B_{\varepsilon}\right\|_{\mathcal{L}(\mathcal{U})} \leqslant M \quad(\varepsilon \in(0, \pi), \text { Re } s=\alpha) .
$$


Let $s=\alpha+i \beta$, with $\alpha>0$ fixed and $\beta \in \mathbb{R}$. For $\varepsilon \in(0, \pi)$ and $u \in \mathcal{U}$ we denote $y_{\varepsilon}=\left(s^{2} I+A_{0}\right)^{-1} B_{\varepsilon} u$. This means that $y_{\varepsilon}$ satisfies the conditions

$$
\begin{gathered}
s^{2} y_{\varepsilon}-\frac{\mathrm{d}^{2} y_{\varepsilon}}{\mathrm{d} x^{2}}=\frac{1}{\sqrt{\varepsilon}} u \chi_{[0, \varepsilon]} \quad(x \in[0, \pi]), \\
\frac{\mathrm{d} y_{\varepsilon}}{\mathrm{d} x}(0)=0, \quad y_{\varepsilon}(\pi)=0 .
\end{gathered}
$$

To estimate $y_{\varepsilon}$ we introduce the associated Green function $K(\xi, x)$ which satisfies, in the distributions sense

$$
\begin{gathered}
s^{2} K-\frac{\partial^{2} K}{\partial x^{2}}=\delta_{\xi}, \\
\frac{\partial K}{\partial x}(\xi, 0)=0, \quad K(\xi, \pi)=0
\end{gathered}
$$

where $\delta_{\xi}$ is the Dirac mass concentrated at $\xi \in(0, \pi)$. It is not difficult to check that

$$
K(\xi, x)= \begin{cases}\frac{\sinh [s(\pi-\xi)]}{s \cosh (s \pi)} \cosh (s x) & \text { if } x<\xi \\ \frac{\cosh (s \xi)}{s \cosh (s \pi)} \sinh [s(\pi-x)] & \text { if } x>\xi\end{cases}
$$

Moreover, it is easily verified that there exist $k_{1}(\gamma), k_{2}(\gamma)>0$ such that

$$
\begin{gathered}
|\sinh [s(\pi-\xi)]|+|\cosh (s x)|+|\cosh (s \xi)|+|\sinh [s(\pi-x)]| \leqslant k_{1}(\gamma) \quad(s \in \mathbb{C}, \operatorname{Re} s=\gamma>0), \\
\min \{|\cosh (s \pi)|,|s \cosh (s \pi)|\} \geqslant k_{2}(\gamma) \quad(s \in \mathbb{C}, \operatorname{Re} s=\gamma),
\end{gathered}
$$

so that

$$
|K(\xi, x)| \leqslant \frac{k_{3}(\gamma)}{|s|} \quad(\operatorname{Re} s=\gamma, \xi, x \in[0, \pi]),
$$

for some constant $k_{3}(\gamma)>0$.

The solution $y_{\varepsilon}$ of $(5.18),(5.19)$ is thus given by

$$
y_{\varepsilon}(x)=\frac{1}{\sqrt{\varepsilon}} \int_{0}^{\varepsilon} K_{\varepsilon}(\xi, x) u(\xi) \mathrm{d} \xi
$$

where the Green function $K_{\varepsilon}$ is given by (5.20). Consequently,

$$
\begin{aligned}
\left\|s B_{\varepsilon}^{*}\left(s^{2} I+A_{0}\right)^{-1} B_{\varepsilon} u\right\|^{2}=\left\|s B_{\varepsilon}^{*} y_{\varepsilon}\right\|^{2}=\frac{|s|^{2}}{\varepsilon^{2}} \int_{0}^{\varepsilon}\left|\int_{0}^{\varepsilon} K_{\varepsilon}(\xi, x) u(\xi) \mathrm{d} \xi\right|^{2} \mathrm{~d} x \\
\leqslant \frac{|s|^{2}}{\varepsilon^{2}} \int_{0}^{\varepsilon}|u(\xi)|^{2} \mathrm{~d} \xi \int_{0}^{\varepsilon} \int_{0}^{\varepsilon}|K(\xi, x)|^{2} \mathrm{~d} \xi \mathrm{d} x .
\end{aligned}
$$

The above estimate and (5.21) imply (5.17) and thus the conclusion of the theorem. 


\section{Dirichlet control of the string equation}

In this section we apply our main abstract result from Theorem 4.1 to approximate the system governed by the string equation with Dirichlet boundary control, i.e., of the system

$$
\begin{array}{rrr}
\ddot{q}_{0}(x, t)+\frac{\partial^{2} q_{0}}{\partial x^{2}}(x, t)=0 & ((x, t) \in(0, \pi) \times[0, \tau]) \\
q_{0}(0, t)=u_{0}(t), & q_{0}(\pi, t)=0 & (t \geqslant 0) \\
q_{0}(x, 0)=f(x), & \dot{q}_{0}(x, 0)=g(x), & x \in(0, \pi),
\end{array}
$$

by a sequence of systems described by the string equation with control distributed in the considered spatial domain. To this aim, we have to face a difficulty which was not present in the case of Neumann boundary conditions: the natural state space for the Dirichlet boundary controlled system is $L^{2}[0, \pi] \times H^{-1}(0, \pi)$ whereas the systems with distributed control are naturally described in the state space $H_{0}^{1}(0, \pi) \times L^{2}[0, \pi]$. Therefore, we begin by giving an alternative formulation of the system described by the string equation with

internal control in the same state space as the one generally used for the string equation with Dirichlet boundary control. To this aim, we need some notation.

Let $\chi_{1} \in \mathcal{D}(-1,1)$, with $\chi_{1}(x) \in[0,1]$ for every $x \in \mathbb{R}$ such that

$$
\chi_{1}(x)= \begin{cases}1 & \text { if }|x| \leqslant \frac{3}{4} \\ 0 & \text { if }|x| \geqslant \frac{5}{4}\end{cases}
$$

and (for later convenience)

$$
\int_{0}^{\infty}\left(\chi_{1}(x)\right)^{2} \mathrm{~d} x+\int_{0}^{1} x^{2}\left|\frac{\mathrm{d} \chi_{1}}{\mathrm{~d} x}(x)\right|^{2} \mathrm{~d} x=1 .
$$

For $\varepsilon>0$ we set

$$
\chi_{\varepsilon}(x)=\chi\left(\frac{x}{\varepsilon}\right) \quad(x \in \mathbb{R}),
$$

so that $\chi_{\varepsilon} \in \mathcal{D}(\mathbb{R})$ satisfies

$$
\chi_{\varepsilon}(x)=\left\{\begin{array}{ll}
1 & \text { if }|x| \leqslant \frac{3 \varepsilon}{4} \\
0 & \text { if }|x| \geqslant \frac{5 \varepsilon}{4}
\end{array},\right.
$$

and $\chi_{\varepsilon}(x) \in[0,1]$ for every $x \in \mathbb{R}$. Moreover, we obviously have

$$
\int_{0}^{\pi} \chi_{\varepsilon}^{2}(x) \mathrm{d} x=\varepsilon \int_{0}^{\pi} \chi_{1}^{2}(x) \mathrm{d} x \leqslant \varepsilon \quad\left(\varepsilon \in\left(0, \frac{4 \pi}{5}\right)\right) .
$$

Using the above notation, we introduce, for each $\varepsilon \in\left(0, \frac{4 \pi}{5}\right)$, the control problem

$$
\begin{aligned}
& \ddot{q}_{\varepsilon}(x, t)+\frac{\partial^{2} q_{\varepsilon}}{\partial x^{2}}(x, t)+\frac{\chi_{\varepsilon}(x)}{\sqrt{\varepsilon}} u_{\varepsilon}(x, t)=0 \quad((x, t) \in(0, \pi) \times[0, \tau]), \\
& q_{\varepsilon}(0, t)=q_{\varepsilon}(\pi, t)=0 \quad(t \geqslant 0) \\
& q_{\varepsilon}(x, 0)=f(x), \quad \dot{q}_{\varepsilon}(x, 0)=g(x) \quad(x \in(0, \pi)),
\end{aligned}
$$

Our result on the singular perturbation of the string equation with Dirichlet boundary controls states as follows: 
Theorem 6.1. Given $\tau \geqslant 2 \pi, f \in L^{2}[0, \pi], g \in H^{-1}(0, \pi)$, there exists a family $\left(u_{\varepsilon}\right)_{\varepsilon \in\left(0, \frac{4 \pi}{5}\right)}$ in $L^{2}\left([0, \tau] ; H^{-1}(0, \pi)\right)$ and $u_{0} \in L^{2}[0, \tau]$ such that

1. For each $\varepsilon \in\left(0, \frac{4 \pi}{5}\right)$ the solution of (6.7)-(6.9) satisfies

$$
q_{\varepsilon}(x, \tau)=0, \quad \dot{q}_{\varepsilon}(x, \tau)=0, \quad x \in[0, \pi]
$$

2. For every $\psi \in L^{2}\left([0, \tau] ; H^{2}(0, \pi) \cap H_{0}^{1}(0, \pi)\right)$ we have

$$
\lim _{\varepsilon \rightarrow 0^{+}} \int_{0}^{\tau} \int_{0}^{\frac{5 \varepsilon}{4}} \frac{\chi_{\varepsilon}(x)}{\sqrt{\varepsilon}} u_{\varepsilon}(x, t) \bar{\psi}(\eta, t) \mathrm{d} t=\int_{0}^{\tau} u_{0}(t) \frac{\partial \bar{\psi}(0, t)}{\partial x} \mathrm{~d} t
$$

3.

$$
\lim _{\varepsilon \rightarrow 0^{+}}\left(\left\|q_{\varepsilon}-q_{0}\right\|_{C\left([0, \tau] ; L^{2}[0, \pi]\right)}+\left\|\dot{q}_{\varepsilon}-\dot{q}_{0}\right\|_{C\left([0, \tau] ; H^{-1}(0, \pi)\right)}\right)=0,
$$

where $q_{0}$ is the solution of (6.1)-(6.3).

Moreover, if $f \in H_{0}^{1}(0, \pi)$ and $g \in L^{2}[0, \pi]$ then the family $\left(u_{\varepsilon}\right)_{\varepsilon \in\left(0, \frac{4 \pi}{5}\right)}$ can be chosen in $L^{2}\left([0, \tau] ; L^{2}[0, \pi]\right)$.

We note that the approximating control problems (6.7)-(6.9) are described using a non standard input space, which is $H^{-1}(0, \pi)$. This choice allows to have an exactly controllable system in the state space $L^{2}[0, \pi] \times H^{-1}(0, \pi)$, thus the same as for the boundary control system (6.1)-(6.3). We also remark that conclusion 2 above describes a weak convergence (in $\left.L^{2}\left([0, \pi] ;\left(H^{2}(0, \pi) \cap H_{0}^{1}(0, \pi)\right)^{\prime}\right)\right)$ of the sequence of controlled inputs $\left\{\frac{\chi_{\varepsilon}}{\sqrt{\varepsilon}} u_{\varepsilon}\right\}$ to a control input corresponding to the limiting problem (6.1)-(6.3). A result similar to our theorem above has been obtained for the wave equation in a spatial domain $\Omega \subset \mathbb{R}^{n}$, with $n \geqslant 1$, in Fabre [9]. This result asserts the weak* convergence (up to the extraction of a subsequence) of the controlled trajectories in $L^{\infty}\left([0, T] ; L^{2}(\Omega)\right)$. Theorem 6.1 asserts that, at least in one space dimension, this convergence is strong and does not require the extraction of a subsequence.

Denote $\mathcal{H}=H^{-1}(0, \pi)$ and let $A_{0}$ be defined by

$$
\begin{gathered}
\mathcal{D}\left(A_{0}\right)=H_{0}^{1}(0, \pi), \\
A_{0} f=-\frac{\mathrm{d}^{2} f}{\mathrm{~d} x^{2}} \quad \forall f \in \mathcal{D}\left(A_{0}\right) .
\end{gathered}
$$

As in the previous sections, $\langle\cdot, \cdot\rangle$ and $\|\cdot\|$ stand for the inner product in $\mathcal{H}$ and for the induced norm, respectively. We denote by $\mathcal{H}_{1}$ and $\mathcal{H}_{\frac{1}{2}}$ the spaces $\mathcal{D}\left(A_{0}\right)$ and $\mathcal{D}\left(A_{0}^{\frac{1}{2}}\right)$, respectively, both endowed with the graph norm and by $\mathcal{H}_{-\frac{1}{2}}$ the dual of $\mathcal{H}_{\frac{1}{2}}$ with respect to the pivot space $\mathcal{H}$. It is known (see, for instance [32, Section 10.3]) that, with the above choice of spaces and operators, we have

$$
\mathcal{H}_{\frac{1}{2}}=L^{2}[0, \pi], \quad \mathcal{H}_{-\frac{1}{2}}=\left[H^{2}(0, \pi) \cap H_{0}^{1}(0, \pi)\right]^{\prime},
$$

where $\left[H^{2}(0, \pi) \cap H_{0}^{1}[0, \pi]\right]^{\prime}$ stands for the dual of $H^{2}(0, \pi) \cap H_{0}^{1}(0, \pi)$ with respect to the pivot space $L^{2}[0, \pi]$. 
To describe the string equation with distributed control as a system in the state space $L^{2}[0, \pi] \times H^{-1}(0, \pi)$ we make a nonstandard choice of the control space by setting

$$
\mathcal{U}=\mathcal{H}=H^{-1}(0, \pi)
$$

We next consider the family of control operators $\left(B_{\varepsilon}\right)_{\varepsilon \in\left(0, \frac{4 \pi}{5}\right)} \subset \mathcal{L}(\mathcal{U}, \mathcal{H})$ defined by

$$
B_{\varepsilon} \mathrm{v}=\frac{1}{\sqrt{\varepsilon}} \chi_{\varepsilon} \mathrm{v} \quad\left(\varepsilon \in\left(0, \frac{4 \pi}{5}\right), \quad \mathrm{v} \in \mathcal{U}\right),
$$

where $\left(\chi_{\varepsilon}\right)$ is the family of $C^{\infty}$ functions defined in (6.5). Note that for $\mathrm{v} \in \mathcal{U}=\mathcal{H}=$ $L^{2}[0, \pi]$ and $g \in \mathcal{H}_{\frac{1}{2}}=L^{2}[0, \pi]$ we have

$$
\begin{aligned}
&\left\langle B_{\varepsilon} \mathrm{v}, g\right\rangle_{-\frac{1}{2}, \frac{1}{2}}=\left\langle B_{\varepsilon} \mathrm{v}, g\right\rangle_{H^{-1}(0, \pi)}=\left\langle\left\langle B_{\varepsilon} \mathrm{v}, A_{0}^{-1} g\right\rangle\right\rangle_{H^{-1}(0, \pi), H_{0}^{1}(0, \pi)} \\
&=\left\langle\left\langle\mathrm{v}, \frac{1}{\sqrt{\varepsilon}} \chi_{\varepsilon} A_{0}^{-1} g\right\rangle\right\rangle_{H^{-1}(0, \pi), H_{0}^{1}(0, \pi)}=\left\langle\mathrm{v}, \frac{1}{\sqrt{\varepsilon}} A_{0}\left(\chi_{\varepsilon} A_{0}^{-1} g\right)\right\rangle_{\mathcal{U}},
\end{aligned}
$$

where $\langle\langle\cdot, \cdot\rangle\rangle$ denotes the duality with respect to the pivot space $L^{2}[0, \pi]$ and, as above $\langle\cdot, \cdot\rangle$ denotes the duality with respect to the pivot space $\mathcal{H}=H^{-1}(0, \pi)$. We thus have

$$
B_{\varepsilon}^{*} g=\frac{1}{\sqrt{\varepsilon}} A_{0}\left(\chi_{\varepsilon} A_{0}^{-1} g\right) \quad\left(\varepsilon \in\left(0, \frac{4 \pi}{5}\right), \quad g \in \mathcal{H}_{\frac{1}{2}}\right) .
$$

For $\varepsilon \in(0, \pi)$ we choose to describe the system (6.7)-(6.9) in the form

$$
\begin{gathered}
\ddot{q}_{\varepsilon}+A_{0} q_{\varepsilon}+B_{\varepsilon} u=0 \\
q(0)=f, \quad \dot{q}(0)=g,[0, \tau])
\end{gathered}
$$

where, as mentioned above, $B_{\varepsilon} \in \mathcal{L}(\mathcal{U}, \mathcal{H})$ for every $\varepsilon \in(0, \pi)$ is defined in (6.11). The boundary control system (6.1)-(6.3) can also be written in the form (6.13)-(6.14) (see, for instance, [32, Section 10.3]) with $\varepsilon=0$ and with the (unbounded) control operator $B_{0} \in \mathcal{L}\left(\mathcal{U}_{0}, \mathcal{H}_{-\frac{1}{2}}\right)$ defined by

$$
B_{0} \mathrm{v}=A_{0} D \mathrm{v} \quad\left(\mathrm{v} \in \mathcal{U}_{0}\right)
$$

where

$$
D \mathrm{v}(x)=\frac{\mathrm{v}}{\pi}(\pi-x) \quad(x \in(0, \pi)) .
$$

It is not difficult to check (see again [32, Section 10.3]) that the adjoint $B_{0}^{*} \in \mathcal{L}\left(\mathcal{H}_{\frac{1}{2}}, \mathcal{U}\right)$ of $B_{0}$ (recall that $\mathcal{U}$ and $\mathcal{H}$ are identified with their duals) is

$$
B_{0}^{*} g=\left.\frac{\mathrm{d}}{\mathrm{d} x}\left(A_{0}^{-1} g\right)\right|_{x=0} \quad\left(g \in \mathcal{H}_{\frac{1}{2}}\right) .
$$

Proposition 6.2. With the above notation, the operators $A_{0}$ and $\left(B_{\varepsilon}\right)_{0<\varepsilon<\frac{4 \pi}{5}}$ satisfy for every $\tau \geqslant 2 \pi$ the assumption (4.5) in Theorem 4.1.

Proof. Let $f \in \mathcal{H}_{\frac{1}{2}}, g \in \mathcal{H}$ and let $\varphi \in C\left([0, \tau] ; \mathcal{H}_{\frac{1}{2}}\right) \cap C^{1}([0, \tau] ; \mathcal{H})$ be the solution of

$$
\ddot{\varphi}(t)+A_{0} \varphi(t)=0, \quad \varphi(0)=f, \quad \dot{\varphi}(0)=g .
$$


Using (6.12) it follows that

$$
\int_{0}^{\tau}\left\|B_{\varepsilon}^{*} \dot{\varphi}\right\|_{\mathcal{U}}^{2} \mathrm{~d} t=\frac{1}{\varepsilon} \int_{0}^{\tau}\left\|A_{0}\left(\chi_{\varepsilon} A_{0}^{-1} \dot{\varphi}(t)\right)\right\|_{\mathcal{U}}^{2} \mathrm{~d} t \quad(\tau \geqslant 0)
$$

Since $\mathcal{U}=\mathcal{H}=H^{-1}(0, \pi)$ and $\mathcal{H}_{\frac{1}{2}}=L^{2}(0, \pi)$, it follows that

$$
\begin{aligned}
& \int_{0}^{\tau}\left\|B_{\varepsilon}^{*} \dot{\varphi}\right\|_{\mathcal{U}}^{2} \mathrm{~d} t=\frac{1}{\varepsilon} \int_{0}^{\tau}\left\|A_{0}^{\frac{1}{2}}\left(\chi_{\varepsilon} A_{0}^{-1} \dot{\varphi}(t)\right)\right\|_{L^{2}[0, \pi]}^{2} \mathrm{~d} t \\
& \left.\left.\quad=\frac{1}{\varepsilon} \int_{0}^{\tau} \int_{0}^{\pi} \mid \frac{\partial}{\partial x}\left(\chi_{\varepsilon} A_{0}^{-1} \dot{\varphi}(t, x)\right)\right)\left.\right|^{2} \mathrm{~d} x \mathrm{~d} t \geqslant \frac{1}{\varepsilon} \int_{0}^{\tau} \int_{0}^{\frac{\varepsilon}{2}} \mid \frac{\partial}{\partial x}\left(A_{0}^{-1} \dot{\varphi}(t, x)\right)\right)\left.\right|^{2} \mathrm{~d} x \mathrm{~d} t .
\end{aligned}
$$

Let

$$
f=\sum_{n \geqslant 1} a_{n} \psi_{n}, \quad g=\sum_{n \geqslant 1} n b_{n} \psi_{n}
$$

where $\left(a_{n}\right),\left(b_{n}\right) \in l^{2}(\mathbb{C})$ and

$$
\psi_{n}(x)=\sqrt{\frac{2}{\pi}} \sin (n x) \quad(n \geqslant 1) .
$$

Note, recalling that $\|\cdot\|$ and $\|\cdot\|_{\frac{1}{2}}$ stand for the norms in $\mathcal{H}=H^{-1}(0, \pi)$ and $\mathcal{H}_{\frac{1}{2}}=L^{2}[0, \pi]$, respectively, that

$$
\|f\|_{\frac{1}{2}}^{2}=\sum_{n \geqslant 1}\left|a_{n}\right|^{2}, \quad\|g\|^{2}=\sum_{n \geqslant 1}\left|b_{n}\right|^{2}
$$

A simple calculation shows that

$$
\left.\int_{0}^{\tau} \int_{0}^{\frac{\varepsilon}{2}} \mid \frac{\partial}{\partial x}\left(A_{0}^{-1} \dot{\varphi}(t, x)\right)\right)\left.\right|^{2} \mathrm{~d} x \mathrm{~d} t=\int_{0}^{\tau} \int_{0}^{\frac{\varepsilon}{2}}\left|\sum_{n \geqslant 1}\left(-a_{n} \sin (n t)+b_{n} \cos (n t)\right) \cos (n x)\right|^{2} \mathrm{~d} x \mathrm{~d} t .
$$

Using Parseval's theorem it follows that for every $\tau \geqslant 2 \pi$ there exists $K_{\tau}>0$ such that

$$
\begin{aligned}
\left.\int_{0}^{\tau} \int_{0}^{\frac{\varepsilon}{2}} \mid \frac{\partial}{\partial x}\left(A_{0}^{-1} \dot{\varphi}(t, x)\right)\right)\left.\right|^{2} \mathrm{~d} x \mathrm{~d} t & \geqslant \frac{2}{\pi} \sum_{n \geqslant 1}\left(\left|a_{n}\right|^{2}+\left|b_{n}\right|^{2}\right) \int_{0}^{\frac{\varepsilon}{2}} \cos ^{2}(n x) \mathrm{d} x \\
& \geqslant \frac{\varepsilon}{3 \pi}\left(\|f\|_{\frac{1}{2}}^{2}+\|g\|^{2}\right)
\end{aligned}
$$

where we have used an elementary inequality similar to (5.16). Therefore combining the last estimate with (6.17) proves (4.5).

Next we verify that the hypothesis (4.7) of Theorem 4.1 is satisfied for the system considered in this section.

Proposition 6.3. With the above notation, for every $f \in \mathcal{H}_{\frac{1}{2}}$ we have

$$
\lim _{\varepsilon \rightarrow 0^{+}} B_{\varepsilon} B_{\varepsilon}^{*} f=B_{0} B_{0}^{*} f \quad \text { in } \quad \mathcal{H}_{-\frac{1}{2}} .
$$


Proof. Let $f, g \in \mathcal{H}_{\frac{1}{2}}$. Using (6.12) we have

$$
\begin{aligned}
\left\langle B_{\varepsilon} B_{\varepsilon}^{*} f, g\right\rangle_{-\frac{1}{2}, \frac{1}{2}}= & \left\langle B_{\varepsilon}^{*} f, B_{\varepsilon}^{*} g\right\rangle_{\mathcal{U}}=\frac{1}{\varepsilon}\left\langle A_{0}\left(\chi_{\varepsilon}\left(A_{0}^{-1} f\right)\right), A_{0}\left(\chi_{\varepsilon}\left(A_{0}^{-1} g\right)\right)\right\rangle_{\mathcal{U}} \\
& =\frac{1}{\varepsilon} \int_{0}^{\pi} A_{0}^{\frac{1}{2}}\left(\chi_{\varepsilon}\left(A_{0}^{-1} f\right)\right) \overline{A_{0}^{\frac{1}{2}}\left(\chi_{\varepsilon}\left(A_{0}^{-1} g\right)\right)} \mathrm{d} x \\
& =\frac{1}{\varepsilon} \int_{0}^{\varepsilon} \frac{\mathrm{d}}{\mathrm{d} x}\left(\chi_{\varepsilon}\left(A_{0}^{-1} f\right)\right) \overline{\frac{\mathrm{d}}{\mathrm{d} x}\left(\chi_{\varepsilon}\left(A_{0}^{-1} g\right)\right)} \mathrm{d} x \quad\left(\varepsilon \in\left(0, \frac{4 \pi}{5}\right)\right) .
\end{aligned}
$$

We clearly have

$$
\begin{aligned}
& \frac{1}{\varepsilon} \int_{0}^{\varepsilon} \frac{\mathrm{d}}{\mathrm{d} x}\left(\chi_{\varepsilon}\left(A_{0}^{-1} f\right)\right) \overline{\frac{\mathrm{d}}{\mathrm{d} x}\left(\chi_{\varepsilon}\left(A_{0}^{-1} g\right)\right)} \mathrm{d} x\left.=\frac{1}{\varepsilon} \int_{0}^{\varepsilon}\left|\frac{\mathrm{d} \chi_{\varepsilon}}{\mathrm{d} x}\right|^{2}\left(A_{0}^{-1} f\right)\right) \overline{\left.\left(A_{0}^{-1} g\right)\right)} \mathrm{d} x \\
&+\frac{1}{\varepsilon} \int_{0}^{\varepsilon} \chi_{\varepsilon} \frac{\mathrm{d} \chi_{\varepsilon}}{\mathrm{d} x}\left(\frac{\mathrm{d}\left(A_{0}^{-1} f\right)}{\mathrm{d} x}\right) \overline{\left.\left(A_{0}^{-1} g\right)\right)} \mathrm{d} x+\frac{1}{\varepsilon} \int_{0}^{\varepsilon} \chi_{\varepsilon} \frac{\mathrm{d} \chi_{\varepsilon}}{\mathrm{d} x}\left(A_{0}^{-1} f\right) \overline{\left(\frac{\mathrm{d}\left(A_{0}^{-1} g\right)}{\mathrm{d} x}\right)} \mathrm{d} x \\
&+\frac{1}{\varepsilon} \int_{0}^{\varepsilon} \chi_{\varepsilon}^{2}\left(\frac{\mathrm{d}\left(A_{0}^{-1} f\right)}{\mathrm{d} x}\right) \overline{\left(\frac{\mathrm{d}\left(A_{0}^{-1} g\right)}{\mathrm{d} x}\right)} \mathrm{d} x
\end{aligned}
$$

For $f, g \in \mathcal{H}_{\frac{1}{2}}=L^{2}[0, \pi]$ we have $A_{0}^{-1} f, A_{0}^{-1} g \in H^{2}(0, \pi) \cap H_{0}^{1}(0, \pi)$. Thus, applying Taylor's formula,

$$
\begin{aligned}
& \left(A_{0}^{-1} f\right)(x)=x \frac{\mathrm{d}\left(A_{0}^{-1} f\right)}{\mathrm{d} x}(0)-\int_{0}^{x}(x-y) f(y) \mathrm{d} y \quad\left(f \in L^{2}[0, \pi], x \in[0, \pi]\right), \\
& \left(A_{0}^{-1} g\right)(x)=x \frac{\mathrm{d}\left(A_{0}^{-1} g\right)}{\mathrm{d} x}(0)-\int_{0}^{x}(x-y) g(y) \mathrm{d} y \quad\left(g \in L^{2}[0, \pi], x \in[0, \pi]\right) .
\end{aligned}
$$

Consequently, there exist $F, G \in L^{\infty}[0, \pi]$ such that

$$
\left(A_{0}^{-1}\right) f(x)-x \frac{\mathrm{d}\left(A_{0}^{-1} f\right)}{\mathrm{d} x}(0)=x^{\frac{3}{2}} F(x), \quad\left(A_{0}^{-1} g\right)(x)-x \frac{\mathrm{d}\left(A_{0}^{-1} g\right)}{\mathrm{d} x}(0)=x^{\frac{3}{2}} G(x),
$$

with

$$
\|F\|_{L^{\infty}[0, \pi]} \leqslant \tilde{K}\|f\|_{\frac{1}{2}}, \quad\|G\|_{L^{\infty}[0, \pi]} \leqslant \tilde{K}\|g\|_{\frac{1}{2}} .
$$

Inserting the above formulas in the first term in the right hand side of (6.20) it follows that

$$
\begin{gathered}
\frac{1}{\varepsilon} \int_{0}^{\varepsilon}\left|\frac{\mathrm{d} \chi_{\varepsilon}}{\mathrm{d} x}\right|^{2}\left(A_{0}^{-1} f\right) \overline{\left(A_{0}^{-1} g\right)} \mathrm{d} x \\
=\frac{1}{\varepsilon}\left(\int_{0}^{\varepsilon} x^{2}\left|\frac{\mathrm{d} \chi_{\varepsilon}}{\mathrm{d} x}\right|^{2} \mathrm{~d} x\right)\left(\frac{\mathrm{d}\left(A_{0}^{-1} f\right)}{\mathrm{d} x}\right)(0) \overline{\left(\frac{\mathrm{d}\left(A_{0}^{-1} g\right)}{\mathrm{d} x}\right)(0)} \\
+R_{\varepsilon}\left[\begin{array}{l}
f \\
g
\end{array}\right],
\end{gathered}
$$

with

$$
\left|R_{\varepsilon}\left[\begin{array}{l}
f \\
g
\end{array}\right]\right| \leqslant C \sqrt{\varepsilon}\|f\|_{\frac{1}{2}}\|g\|_{\frac{1}{2}} \quad\left(\varepsilon \in\left(0, \frac{4 \pi}{5}\right), f, g \in \mathcal{H}_{\frac{1}{2}}\right),
$$

and $C$ an absolute constant. 
Concerning the first term in the right had side of (6.24) we can use (6.21), (6.22) to obtain that

$$
\begin{gathered}
\frac{1}{\varepsilon}\left(\int_{0}^{\varepsilon} x^{2}\left|\frac{\mathrm{d} \chi_{\varepsilon}}{\mathrm{d} x}(x)\right|^{2} \mathrm{~d} x\right)\left(\frac{\mathrm{d}\left(A_{0}^{-1} f\right)}{\mathrm{d} x}\right)(0) \overline{\left(\frac{\mathrm{d}\left(A_{0}^{-1} g\right)}{\mathrm{d} x}\right)(0)} \\
=\frac{1}{\varepsilon}\left(\frac{\mathrm{d}\left(A_{0}^{-1} f\right)}{\mathrm{d} x}\right)(0) \overline{\left(\frac{\mathrm{d}\left(A_{0}^{-1} g\right)}{\mathrm{d} x}\right)(0)} \int_{0}^{\varepsilon} x^{2}\left|\frac{\mathrm{d} \chi_{\varepsilon}}{\mathrm{d} x}(x)\right|^{2} \mathrm{~d} x+\tilde{R}_{\varepsilon}\left[\begin{array}{c}
f \\
g
\end{array}\right] \\
=\left(\frac{\mathrm{d}\left(A_{0}^{-1} f\right)}{\mathrm{d} x}\right)(0) \overline{\left(\frac{\mathrm{d}\left(A_{0}^{-1} g\right)}{\mathrm{d} x}\right)(0)} \int_{0}^{1} y^{2}\left|\frac{\mathrm{d} \chi_{1}}{\mathrm{~d} x}(y)\right|^{2} \mathrm{~d} y+\tilde{R}_{\varepsilon}\left[\begin{array}{l}
f \\
g
\end{array}\right],
\end{gathered}
$$

where

$$
\left|\tilde{R}_{\varepsilon}\left[\begin{array}{l}
f \\
g
\end{array}\right]\right| \leqslant \tilde{C}\|f\|_{\frac{1}{2}}\|g\|_{\frac{1}{2}} \quad\left(\varepsilon \in\left(0, \frac{4 \pi}{5}\right), f, g \in \mathcal{H}_{\frac{1}{2}}\right) .
$$

The last two estimates, combined with (6.24) and (6.25), imply that the first term in the right hand side of (6.20) satisfies

$$
\begin{aligned}
\left.\left|\frac{1}{\varepsilon} \int_{0}^{\varepsilon}\right| \frac{\mathrm{d} \chi_{\varepsilon}}{\mathrm{d} x}\right|^{2}\left(A_{0}^{-1} f\right) \overline{\left.\left(A_{0}^{-1} g\right)\right)} \mathrm{d} x & -\left(\frac{\mathrm{d}\left(A_{0}^{-1} f\right)}{\mathrm{d} x}\right)(0) \overline{\left(\frac{\mathrm{d}\left(A_{0}^{-1} g\right)}{\mathrm{d} x}\right)(0)} \int_{0}^{1} y^{2}\left|\frac{\mathrm{d} \chi_{1}}{\mathrm{~d} x}(y)\right|^{2} \mathrm{~d} y \mid \\
& \leqslant K_{1} \varepsilon\|f\|_{\frac{1}{2}}\|g\|_{\frac{1}{2}}\left(\varepsilon \in\left(0, \frac{4 \pi}{5}\right), f, g \in \mathcal{H}_{\frac{1}{2}}\right), \quad(6.26)
\end{aligned}
$$

where $K_{1}$ is an absolute constant.

To estimate the second term in the right hand side of (6.20) we remark that, using (6.23) and the inequality $\left\|\frac{\mathrm{d}\left(A_{0}^{-1} f\right)}{\mathrm{d} x}\right\|_{L^{\infty}[0, \pi]} \leqslant\|f\|_{\frac{1}{2}}$, we have

$$
\begin{aligned}
\frac{1}{\varepsilon}\left|\int_{0}^{\varepsilon} \chi_{\varepsilon} \frac{\mathrm{d} \chi_{\varepsilon}}{\mathrm{d} x}\left(\frac{\mathrm{d}\left(A_{0}^{-1} f\right)}{\mathrm{d} x}\right) \overline{\left.\left(A_{0}^{-1} g\right)\right)} \mathrm{d} x\right| & \leqslant \frac{K_{3}}{\varepsilon^{2}} \int_{0}^{\varepsilon}\left|\frac{\mathrm{d}\left(A_{0}^{-1} f\right)}{\mathrm{d} x}\right|\left|A_{0}^{-1} g\right| \mathrm{d} x \\
\leqslant & \frac{K_{4}\|g\|_{\frac{1}{2}}}{\varepsilon^{2}} \int_{0}^{\varepsilon} x^{\frac{3}{2}}\left|\frac{\mathrm{d}\left(A_{0}^{-1} f\right)}{\mathrm{d} x}\right| \mathrm{d} x \leqslant K_{5} \sqrt{\varepsilon}\|f\|_{\frac{1}{2}}\|g\|_{\frac{1}{2}} \quad\left(f, g \in \mathcal{H}_{\frac{1}{2}}\right) .
\end{aligned}
$$

Similarly, the third term in the right hand side of (6.20) satisfies

$$
\frac{1}{\varepsilon}\left|\int_{0}^{\varepsilon} \chi_{\varepsilon} \frac{\mathrm{d} \chi_{\varepsilon}}{\mathrm{d} x}\left(A_{0}^{-1} f\right) \overline{\left(\frac{\mathrm{d}\left(A_{0}^{-1} g\right)}{\mathrm{d} x}\right)} \mathrm{d} x\right| \leqslant K_{6} \sqrt{\varepsilon}\|f\|_{\frac{1}{2}}\|g\|_{\frac{1}{2}} \quad\left(f, g \in \mathcal{H}_{\frac{1}{2}}\right) .
$$

To estimate the last term in the right hand side of (6.20) we remark that

$$
\begin{array}{ll}
\frac{\mathrm{d}\left(A_{0}^{-1} f\right)}{\mathrm{d} x}(x)=\frac{\mathrm{d}\left(A_{0}^{-1} f\right)}{\mathrm{d} x}(0)+x \tilde{f}(x) & \left(f \in \mathcal{H}_{\frac{1}{2}}, x \in[0, \pi]\right), \\
\frac{\mathrm{d}\left(A_{0}^{-1} g\right)}{\mathrm{d} x}(x)=\frac{\mathrm{d}\left(A_{0}^{-1} g\right)}{\mathrm{d} x}(0)+x \tilde{g}(x) & \left(g \in \mathcal{H}_{\frac{1}{2}}, x \in[0, \pi]\right),
\end{array}
$$

with $\tilde{f}, \tilde{g} \in L^{1}[0, \pi]$ satisfying

$$
\tilde{f}(x) \leqslant \frac{\tilde{K}}{\sqrt{x}}\|f\|_{\frac{1}{2}}, \quad \tilde{g}(x) \leqslant \frac{\tilde{K}}{\sqrt{x}}\|g\|_{\frac{1}{2}} \quad(x \in(0, \pi]),
$$


for some absolute constant $\tilde{K}>0$. It follows that

$$
\begin{aligned}
& \frac{1}{\varepsilon} \int_{0}^{\varepsilon} \chi_{\varepsilon}^{2}\left(\frac{\mathrm{d}\left(A_{0}^{-1} f\right)}{\mathrm{d} x}\right) \overline{\left(\frac{\mathrm{d}\left(A_{0}^{-1} g\right)}{\mathrm{d} x}\right)} \mathrm{d} x \\
& =\frac{1}{\varepsilon} \int_{0}^{\varepsilon} \chi_{\varepsilon}^{2}(x)\left(\frac{\mathrm{d}\left(A_{0}^{-1} f\right)}{\mathrm{d} x}(0)+x \tilde{f}(x)\right) \overline{\left(\frac{\mathrm{d}\left(A_{0}^{-1} g\right)}{\mathrm{d} x}(0)+x \tilde{g}(x)\right)} \mathrm{d} x \\
& =\frac{1}{\varepsilon} \frac{\mathrm{d}\left(A_{0}^{-1} f\right)}{\mathrm{d} x}(0) \overline{\frac{\mathrm{d}\left(A_{0}^{-1} g\right)}{\mathrm{d} x}(0)} \int_{0}^{\varepsilon} \chi_{\varepsilon}^{2}(x) \mathrm{d} x+\frac{1}{\varepsilon} \frac{\mathrm{d}\left(A_{0}^{-1} f\right)}{\mathrm{d} x}(0) \int_{0}^{\varepsilon} x \chi_{\varepsilon}^{2}(x) \tilde{g}(x) \mathrm{d} x \\
& +\frac{1}{\varepsilon} \overline{\frac{\mathrm{d}\left(A_{0}^{-1} g\right)}{\mathrm{d} x}(0)} \int_{0}^{\varepsilon} x \chi_{\varepsilon}^{2}(x) \tilde{f}(x) \mathrm{d} x+\frac{1}{\varepsilon} \int_{0}^{\varepsilon} x^{2} \chi_{\varepsilon}^{2}(x) \tilde{f}(x) \tilde{g}(x) \mathrm{d} x .
\end{aligned}
$$

Using the fact that $\int_{0}^{\varepsilon} \chi_{\varepsilon}^{2}(x) \mathrm{d} x=\varepsilon \int_{0}^{\pi} \chi_{1}^{2}(x) \mathrm{d} x$, together with (6.29) it follows that

$$
\begin{gathered}
\left|\frac{1}{\varepsilon} \int_{0}^{\varepsilon} \chi_{\varepsilon}^{2}\left(\frac{\mathrm{d}\left(A_{0}^{-1} f\right)}{\mathrm{d} x}\right) \overline{\left(\frac{\mathrm{d}\left(A_{0}^{-1} g\right)}{\mathrm{d} x}\right)} \mathrm{d} x-\frac{\mathrm{d}\left(A_{0}^{-1} f\right)}{\mathrm{d} x}(0) \overline{\frac{\mathrm{d}\left(A_{0}^{-1} g\right)}{\mathrm{d} x}(0)} \int_{0}^{\pi} \chi_{1}^{2}(x) \mathrm{d} x\right| \\
\leqslant \widetilde{K} \sqrt{\varepsilon}\|f\|_{\frac{1}{2}}\|g\|_{\frac{1}{2}} \quad\left(f, g \in \mathcal{H}_{\frac{1}{2}}\right),
\end{gathered}
$$

for some universal constant $\widetilde{K}$. By combining the last estimate with (6.26), (6.27) and (6.28) we obtain the conclusion (6.18).

We are now in a position to prove the main result in this section.

Proof of Theorem 6.1. We have seen in Propositions 6.2 and 6.3 that the operators $A_{0}$ and $\left(B_{\varepsilon}\right)_{0<\varepsilon<\frac{4 \pi}{5}}$ satisfy for every $\tau \geqslant 2 \pi$ the assumptions (4.5) and (4.7) of Theorem 4.1. We show below that $A_{0}$ and $B_{\varepsilon}$ also satisfy, for every $\varepsilon \in\left(0, \frac{4 \pi}{5}\right), \gamma>0$ and $s \in \mathbb{C}, \operatorname{Re} s=\gamma$ the assumption (4.6) in the same theorem.

To this aim, let $s=\gamma+i \beta$, with $\gamma>0$ fixed and $\beta \in \mathbb{R}$. For $\varepsilon>0$ and $u \in \mathcal{U}$ we denote $y_{\varepsilon}=\left(s^{2} I+A_{0}\right)^{-1} B_{\varepsilon} u$. This means that $y_{\varepsilon}$ satisfies the conditions

$$
\begin{aligned}
s^{2} y_{\varepsilon}-\frac{\mathrm{d}^{2} y_{\varepsilon}}{\mathrm{d} x^{2}} & =\frac{1}{\sqrt{\varepsilon}} \chi_{\varepsilon} u \quad(\text { in } \mathcal{H}), \\
y_{\varepsilon}(0) & =0, \quad y_{\varepsilon}(\pi)=0 .
\end{aligned}
$$

To estimate $y_{\varepsilon}$ we introduce the associated Green function $\mathrm{K}(s ; \xi, x)$ which satisfies

$$
\begin{gathered}
s^{2} \mathrm{~K}-\frac{\partial^{2} \mathrm{~K}}{\partial x^{2}}=\delta_{\xi}, \\
\mathrm{K}(s ; \xi, 0)=0, \quad \mathrm{~K}(s ; \xi, \pi)=0 .
\end{gathered}
$$

We obviously have

$$
\mathrm{K}(s ; \xi, x)= \begin{cases}c_{1} \sinh (s x) & x<\xi, \\ c_{2} \sinh [s(\pi-x)] & x>\xi\end{cases}
$$

with the constants $c_{1}$ and $c_{2}$ to be determined from the conditions

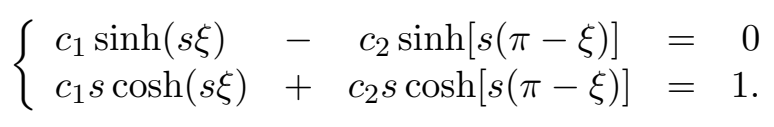


A standard calculation shows that

$$
\mathrm{K}(s ; \xi, x)= \begin{cases}\frac{\sinh [s(\pi-\xi)]}{s \sinh (s \pi)} \sinh (s x) & x<\xi \\ \frac{\sinh (s \xi)}{s \sinh (s \pi)} \sinh [s(\pi-x)] & x>\xi\end{cases}
$$

It is not difficult to show that there exist $k_{1}(\alpha), k_{2}(\alpha)>0$ such that

$$
\begin{gathered}
|\sinh [s(\pi-\xi)]|+|\cosh (s x)|+|\cosh (s \xi)|+|\sinh [s(\pi-x)]| \leqslant k_{1}(\alpha) \quad(s \in \mathbb{C}, \operatorname{Re} s=\alpha), \\
\min \{|\cosh (s \pi)|,|s \cosh (s \pi)|\} \geqslant k_{2}(\alpha) \quad(s \in \mathbb{C}, \operatorname{Re} s=\alpha),
\end{gathered}
$$

so that

$$
|\mathrm{K}(s ; \xi, x)| \leqslant \frac{k_{3}(\alpha)}{|s|} \quad(\operatorname{Re} s=\alpha, \xi, x \in[0, \pi]),
$$

for some constant $k_{3}(\alpha)>0$.

The solution $y_{\varepsilon}$ of $(6.30),(6.31)$ is thus given by

$$
y_{\varepsilon}(x)=\frac{1}{\varepsilon^{1 / 2}}\left\langle\chi_{\varepsilon} u, \mathrm{~K}(s ; \cdot, x)\right\rangle_{H^{-1}(0, \pi), H_{0}^{1}(0, \pi)}=\frac{1}{\varepsilon^{1 / 2}}\left\langle u, \chi_{\varepsilon} \mathrm{K}(s ; \cdot, x)\right\rangle_{H^{-1}(0, \pi), H_{0}^{1}(0, \pi)} .
$$

where the Green's function $\mathrm{K}$ is given by (6.33).

Consequently, using (6.12), we have

$$
s B_{\varepsilon}^{*}\left(s^{2} I+A_{0}\right)^{-1} B_{\varepsilon} u=s B_{\varepsilon}^{*} y_{\varepsilon}=\frac{s}{\sqrt{\varepsilon}} A_{0}\left(\chi_{\varepsilon} \tilde{y}_{\varepsilon}\right),
$$

where

$$
\tilde{y}_{\varepsilon}(x)=\left(A_{0}^{-1} y_{\varepsilon}\right)(x) \quad\left(\varepsilon, x \in\left(0, \frac{4 \pi}{5}\right)\right) .
$$

To prove that estimate (4.6) holds, and hence complete the proof of Theorem 6.1 , we need to show that the terms in (6.36) are bounded in $H^{-1}(0, \pi)$, or equivalently, that there exists $d_{\gamma}>0$ for which

$$
\left\|\chi_{\varepsilon} \tilde{y}_{\varepsilon}\right\|_{H^{1}(0, \pi)}^{2} \leq d_{\gamma} \frac{\varepsilon}{s^{2}}\|u\|_{H^{-1}(0, \pi)}^{2}, \quad \varepsilon \in\left(0, \frac{4 \varepsilon}{5}\right), u \in H^{-1}(0, \pi) .
$$

It is not difficult to verify that

$$
\tilde{\mathrm{K}}(s ; \xi, x):=\left(A_{0}^{-1} \mathrm{~K}\right)(s ; \cdot, x)= \begin{cases}-\frac{\mathrm{K}(s ; \xi, x)}{s^{2}}+\frac{\xi}{\pi s^{2}}(\pi-x) & \xi<x \\ -\frac{\mathrm{K}(s ; \xi, x)}{s^{2}}+\frac{\pi-\xi}{\pi s^{2}} x & \xi>x .\end{cases}
$$

Therefore (6.35) and some calculations imply that

$$
\tilde{y}_{\varepsilon}(x)=\frac{1}{\varepsilon^{1 / 2}}\left\langle u, \chi_{\varepsilon} \tilde{\mathrm{K}}(s ; \cdot, x)\right\rangle_{H^{-1}(0, \pi), H_{0}^{1}(0, \pi)} .
$$

The last two formulas imply that

$$
\left|\tilde{y}_{\varepsilon}(x)\right| \leqslant \frac{1}{\sqrt{\varepsilon}}\|u\|_{H^{-1}(0, \pi)}\left\|\chi_{\varepsilon}(\cdot) \tilde{\mathrm{K}}(s ; \cdot, x)\right\|_{H_{0}^{1}(0, \pi)} .
$$


Using (6.33) and (6.23) together with standard estimates, there exists $c_{\gamma}>0$ such that

$$
\begin{aligned}
& \left\|\chi_{\varepsilon}(\cdot) \tilde{\mathrm{K}}(s ; \cdot, x)\right\|_{H_{0}^{1}(0, \pi)}^{2}=\int_{0}^{\varepsilon}\left|\frac{\partial}{\partial \xi}\left(\chi_{\varepsilon}(\xi) \tilde{\mathrm{K}}(s ; \xi, x)\right)\right|^{2} \mathrm{~d} \xi \\
& \leqslant 2 \int_{0}^{\varepsilon}\left|\frac{\mathrm{d} \chi_{\varepsilon}}{\mathrm{d} \xi}(\xi)\right|^{2}|\tilde{\mathrm{K}}(s ; \xi, x)|^{2} \mathrm{~d} \xi+2 \int_{0}^{\varepsilon}\left|\chi_{\varepsilon}(\xi)\right|^{2}\left|\frac{\partial \tilde{\mathrm{K}}}{\partial \xi}(s ; \xi, x)\right|^{2} \mathrm{~d} \xi \\
& \leqslant \frac{2}{\varepsilon^{2}} \int_{0}^{\varepsilon}\left|\frac{\partial^{2} \tilde{\mathrm{K}}}{\partial \xi^{2}}(s ; \xi, x)\right|^{2} \mathrm{~d} \xi \int_{0}^{\varepsilon} \xi^{3} \mathrm{~d} \xi+2 \int_{0}^{\varepsilon}\left|\frac{\partial \tilde{\mathrm{K}}}{\partial \xi}(s ; \xi, x)\right|^{2} \mathrm{~d} \xi \\
& \leqslant c_{\gamma} \frac{\varepsilon^{3}}{|s|^{2}}+2 \int_{0}^{\varepsilon}\left|\frac{\partial \tilde{\mathrm{K}}}{\partial \xi}(s ; \xi, x)\right|^{2} \mathrm{~d} \xi .
\end{aligned}
$$

Therefore

$$
\left|\tilde{y}_{\varepsilon}(x)\right|^{2} \leqslant\|u\|_{H^{-1}(0, \pi)}^{2}\left(c_{\gamma} \frac{\varepsilon^{2}}{|s|^{2}}+\frac{2}{\varepsilon} \int_{0}^{\varepsilon}\left|\frac{\partial \tilde{\mathrm{K}}}{\partial \xi}(s ; \xi, x)\right|^{2} \mathrm{~d} \xi\right) .
$$

On the other hand (6.40) implies that

$$
\frac{\mathrm{d} \tilde{y}_{\varepsilon}}{\mathrm{d} x}(x)=\frac{1}{\varepsilon^{1 / 2}}\left\langle u, \chi_{\varepsilon}(\cdot) \frac{\partial \tilde{\mathrm{K}}}{\partial x}(s ; \cdot, x)\right\rangle_{H^{-1}(0, \pi), H_{0}^{1}(0, \pi)} .
$$

From (6.39) we have

$$
\begin{gathered}
\frac{\partial \tilde{\mathrm{K}}}{\partial x}(s ; \xi, x)=\left\{\begin{array}{cc}
-\frac{\frac{\partial \mathrm{K}}{\partial x}(s ; \xi, x)}{s^{2}}-\frac{\xi}{\pi s^{2}} & \xi<x \\
-\frac{\partial \mathrm{Jx}(s ; \xi, x)}{s^{2}}+\frac{\pi-\xi}{\pi s^{2}} & \xi>x,
\end{array}\right. \\
\frac{\partial^{2} \tilde{\mathrm{K}}}{\partial x \partial \xi}(s ; \xi, x)= \begin{cases}-\frac{\partial^{2} \mathrm{~K}}{\partial x \partial \xi ; \xi, x)}-\frac{1}{\pi s^{2}} & \xi<x \\
-\frac{\frac{\partial^{2} \mathrm{~K}}{\partial x \partial \xi}(s ; \xi, x)}{s^{2}}-\frac{1}{\pi s^{2}} & \xi>x .\end{cases}
\end{gathered}
$$

The last two formulas imply that there exists $k_{\gamma}>0$ for which

$$
\begin{aligned}
&\left\|\chi_{\varepsilon}(\cdot) \frac{\partial \tilde{\mathrm{K}}}{\partial x}(s ; \cdot, x)\right\|_{H_{0}^{1}(0, \pi)}^{2}=\int_{0}^{\varepsilon}\left|\frac{\partial}{\partial \xi}\left(\chi_{\varepsilon}(\xi) \frac{\partial \tilde{\mathrm{K}}}{\partial x}(s ; \xi, x)\right)\right|^{2} \mathrm{~d} \xi \\
& \leqslant 2 \int_{0}^{\varepsilon}\left|\frac{\partial \chi_{\varepsilon}}{\partial \xi}\right|^{2}\left|\frac{\partial \tilde{\mathrm{K}}}{\partial x}(s ; \xi, x)\right|^{2} \mathrm{~d} \xi+2 \int_{0}^{\varepsilon} \chi_{\varepsilon}^{2}(\xi)\left|\frac{\partial^{2} \tilde{\mathrm{K}}}{\partial x \partial \xi}(s ; \xi, x)\right|^{2} \mathrm{~d} \xi \\
& \leqslant \frac{2}{\varepsilon^{2}} \int_{0}^{\varepsilon} \xi \mathrm{d} \xi \int_{0}^{\varepsilon}\left|\frac{\partial^{2} \tilde{\mathrm{K}}}{\partial x \partial \xi}(s ; \xi, x)\right|^{2} \mathrm{~d} \xi+2 \int_{0}^{\varepsilon}\left|\frac{\partial^{2} \tilde{\mathrm{K}}}{\partial x \partial \xi}(s ; \xi, x)\right|^{2} \mathrm{~d} \xi \\
& \leqslant k_{\gamma} \frac{\varepsilon}{|s|^{2}} .
\end{aligned}
$$

The above estimate and (6.42) imply that

$$
\left|\frac{\mathrm{d} \tilde{y}_{\varepsilon}}{\mathrm{d} x}(x)\right|^{2} \leqslant \frac{k_{\gamma}}{|s|^{2}}\|u\|_{H^{-1}(0, \pi)}^{2} .
$$


Using (6.41) and (6.45) we have

$$
\begin{aligned}
&\left\|\chi_{\varepsilon} \tilde{y}_{\varepsilon}\right\|_{H_{0}^{1}(0, \pi)}^{2}= \int_{0}^{\varepsilon}\left|\frac{\partial}{\partial x}\left(\chi_{\varepsilon}(x) \tilde{y}_{\varepsilon}(x)\right)\right|^{2} \mathrm{~d} x=\int_{0}^{\varepsilon}\left|\frac{\partial \chi_{e}}{\partial x} \tilde{y}_{\varepsilon}(x)+\chi_{\varepsilon}(x) \frac{\mathrm{d} y_{\varepsilon}}{\mathrm{d} x}(x)\right|^{2} \mathrm{~d} x \\
& \leqslant 2 \int_{0}^{\varepsilon}\left|\frac{\partial \chi_{e}}{\partial x}\right|^{2} \tilde{y}_{\varepsilon}^{2}(x) \mathrm{d} x+2 \int_{0}^{\varepsilon} \chi_{\varepsilon}^{2}(x)\left|\frac{\mathrm{d} y_{\varepsilon}}{\mathrm{d} x}(x)\right|^{2} \mathrm{~d} x \\
& \leqslant \frac{2}{\varepsilon^{2}}\left(\int_{0}^{\varepsilon} x \mathrm{~d} x\right)\left(\int_{0}^{\varepsilon}\left|\frac{\mathrm{d} \tilde{y}_{\varepsilon}}{\mathrm{d} x}(x)\right|^{2} \mathrm{~d} x\right)+2 \int_{0}^{\varepsilon}\left|\frac{\mathrm{d} y_{\varepsilon}}{\mathrm{d} x}(x)\right|^{2} \mathrm{~d} x \leqslant \frac{\varepsilon d_{\gamma}}{|s|^{2}}\|u\|_{H^{-1}(0, \pi)}^{2},
\end{aligned}
$$

which establishes estimate (6.38). Thus the conclusions 1, 2, 3, of Theorem 4.1 can be applied to Theorem 6.1. From this it is clear that conclusions 1 and 3 of Theorem 6.1 hold.

To complete the proof, we verify that conclusion 2 of Theorem 4.1 implies conclusion 2, i.e., (6.10) of Theorem 6.1. Given and $\varphi \in L^{2}[0, \pi]$ let $\psi \in H^{2}(0,1) \cap H_{0}^{1}(0,1)$ be the solution of $A_{0} \psi=\varphi$. For $\varepsilon \in(0, \pi)$ we have, recalling that dualities are taken with respect to the the pivot space is $\mathcal{H}=H^{-1}(0, \pi)$,

$$
\begin{aligned}
& \left\langle B_{\varepsilon} u_{\varepsilon}, \varphi\right\rangle_{L^{2}\left([0, \tau] ; \mathcal{H}_{-\frac{1}{2}}\right), L^{2}\left([0, \tau] ; \mathcal{H}_{\frac{1}{2}}\right)}=\int_{0}^{\tau}\left\langle A_{0}^{-\frac{1}{2}} B_{\varepsilon} u_{\varepsilon}, A_{0}^{-\frac{1}{2}} \varphi\right\rangle_{L^{2}[0, \pi]} \mathrm{d} t \\
& =\int_{0}^{\tau}\left\langle B_{\varepsilon} u_{\varepsilon}, A_{0}^{-1} \varphi\right\rangle_{L^{2}[0, \pi]} \mathrm{d} t=\frac{1}{\sqrt{\varepsilon}} \int_{0}^{\tau} \int_{0}^{\pi} \chi_{\varepsilon} u_{\varepsilon} A_{0}^{-1} \bar{\varphi} \mathrm{d} x \mathrm{~d} t=\frac{1}{\sqrt{\varepsilon}} \int_{0}^{\tau} \int_{0}^{\frac{5 \varepsilon}{4}} \chi_{\varepsilon} u_{\varepsilon} \bar{\psi} \mathrm{d} x \mathrm{~d} t .
\end{aligned}
$$

On the other hand, using (6.16) we obtain

$$
\begin{aligned}
\left\langle B_{0} u_{0}, \varphi\right\rangle_{L^{2}\left([0, \tau] ; \mathcal{H}_{-\frac{1}{2}}\right), L^{2}\left([0, \tau] ; \mathcal{H}_{\frac{1}{2}}\right)} & =\left\langle u_{0}, B_{0}^{*} \varphi\right\rangle_{L^{2}[0, \tau]} \\
& =\left.\int_{0}^{\tau} u_{0}(t) \frac{\partial}{\partial x}\left(A_{0}^{-1} \bar{\varphi}\right)\right|_{x=0} \mathrm{~d} t=\int_{0}^{\tau} u_{0}(t) \frac{\partial \bar{\psi}}{\partial x}(t, 0) \mathrm{d} t,
\end{aligned}
$$

which concludes the proof of (6.10).

We still have to check that if $f \in H_{0}^{1}(0, \pi)$ and $g \in L^{2}[0, \pi]$ then the family $\left(u_{\varepsilon}\right)_{\varepsilon \in\left(0, \frac{4 \pi}{5}\right)}$ is contained in $L^{2}\left([0, \tau] ; L^{2}[0, \pi]\right)$. To accomplish this goal we first note that, with $\mathcal{H}$ and $A_{0}$ chosen as at the beginning of this section we have $\mathcal{H}_{\frac{3}{2}}=H^{2}(0, \pi) \cap H_{0}^{1}(0, \pi)$ and $\mathcal{H}_{1}=H_{0}^{1}(0, \pi)$. Moreover, for $\varepsilon>0$ we clearly have $B_{\varepsilon} B_{e}^{*} \in \mathcal{L}\left(\mathcal{H}_{1}, \mathcal{H}_{\frac{1}{2}}\right)$. Thus, according to Remark 2.6 in [4] it follows that $B_{\varepsilon} u_{\varepsilon} \in C\left([0, \tau] ; \mathcal{H}_{\frac{1}{2}}\right)$. Recalling that $\mathcal{H}_{\frac{1}{2}}=L^{2}[0, \pi]$ this implies indeed that $u_{\varepsilon} \in L^{2}\left([0, \tau] ; L^{2}[0, \pi]\right)$ for every $\varepsilon>0$.

\section{Approximation by finite dimensional systems}

In this section we come back to the assumptions and notation in Section 2, i.e., we consider the system (2.6)-(2.7) with a bounded input operator, i.e., with $B_{0} \in \mathcal{L}(\mathcal{U}, \mathcal{H})$. Our aim here is to provide a method for the effective approximation of the control constructed, via Russell's principle, using a family of finite dimensional control systems. To construct this family we need some notation. 
Assume that there exists family $\left(V_{h}\right)_{h>0}$ of finite dimensional subspaces of $\mathcal{H}_{\frac{1}{2}}$ and that there exist $\theta>0, h^{*}>0, C_{0}>0$ such that, for every $h \in\left(0, h^{*}\right)$,

$$
\begin{array}{ll}
\left\|\pi_{h} \varphi-\varphi\right\|_{\frac{1}{2}} \leqslant C_{0} h^{\theta}\|\varphi\|_{1} \quad & \left(\varphi \in \mathcal{H}_{1}\right), \\
\left\|\pi_{h} \varphi-\varphi\right\| \leq C_{0} h^{\theta}\|\varphi\|_{\frac{1}{2}} & \left(\varphi \in \mathcal{H}_{\frac{1}{2}}\right),
\end{array}
$$

where $\pi_{h}$ is the orthogonal projector from $\mathcal{H}_{\frac{1}{2}}$ onto $V_{h}$. Assumptions (7.1)-(7.2) are, in particular, satisfied when finite elements are used for the approximation of Sobolev spaces. The inner product in $V_{h}$ is the restriction of the inner product on $\mathcal{H}$ and it is still denoted by $\langle\cdot, \cdot\rangle$. We define the linear operator $A_{0 h} \in \mathcal{L}\left(V_{h}\right)$ by

$$
\left\langle A_{0 h} \varphi_{h}, \psi_{h}\right\rangle=\left\langle A_{0}^{\frac{1}{2}} \varphi_{h}, A_{0}^{\frac{1}{2}} \psi_{h}\right\rangle \quad\left(\varphi_{h}, \psi_{h} \in V_{h}\right) .
$$

The operator $A_{0 h}$ is clearly symmetric and strictly positive.

Denote $U_{h}=B_{0}^{*} V_{h} \subset \mathcal{U}$ and define the operators $B_{0 h} \in \mathcal{L}(\mathcal{U}, \mathcal{H})$ by

$$
B_{0 h} u=\tilde{\pi}_{h} B_{0} u \quad(u \in U),
$$

where $\tilde{\pi}_{h}$ is the orthogonal projection of $\mathcal{H}$ onto $V_{h}$. Note that $\operatorname{Ran} B_{0 h} \subset V_{h}$. As well-known, since it is an orthogonal projector, the operator $\widetilde{\pi}_{h} \in \mathcal{L}(\mathcal{H})$ is self-adjoint. Moreover, from (7.2) we deduce that

$$
\left\|\varphi-\widetilde{\pi}_{h} \varphi\right\| \leqslant\left\|\varphi-\pi_{h} \varphi\right\| \leqslant C_{0} h^{\theta}\|\varphi\|_{\frac{1}{2}} \quad\left(\varphi \in \mathcal{H}_{\frac{1}{2}}\right) .
$$

The adjoint $B_{0 h}^{*} \in \mathcal{L}(\mathcal{H}, \mathcal{U})$ of $B_{0 h}$ is

$$
B_{0 h}^{*} \varphi=B_{0}^{*} \widetilde{\pi}_{h} \varphi \quad(\varphi \in \mathcal{H}) .
$$

Since $U_{h}=B_{0}^{*} V_{h}$, from (7.6) it follows that $\operatorname{Ran} B_{0 h}^{*}=U_{h}$ and that

$$
\left\langle B_{0 h}^{*} \varphi_{h}, B_{0 h}^{*} \psi_{h}\right\rangle_{\mathcal{U}}=\left\langle B_{0}^{*} \varphi_{h}, B_{0}^{*} \psi_{h}\right\rangle_{\mathcal{U}} \quad\left(\varphi_{h}, \psi_{h} \in V_{h}\right) .
$$

The above assumptions imply that, for every $h^{*}>0$, the family $\left(\left\|B_{0 h}\right\|_{\mathcal{L}(\mathcal{U}, \mathcal{H})}\right)_{h \in\left(0, h^{*}\right)}$ is bounded.

A methodology which seems natural in view of approximating the controls constructed in Section 2 by finite dimensional ones seems to consist in applying Russell's principle to the family of finite dimensional systems obtained from (2.6)-(2.7) by replacing $A_{0}$ by $A_{0 h}$ and $B_{0}$ by $B_{0 h}$. However, as in the case of HUM type controls, there exists at least one initial state in $\mathcal{H}_{\frac{1}{2}} \times \mathcal{H}$ for which the corresponding controls, say $\left(u_{h}\right)_{h>0}$ are not bounded in $L^{2}([0, \tau] ; \mathcal{U})$. This is due to the presence of spurious high frequencies, which implies that the closed-loop systems are not uniformly (with respect to $h$ ) exponentially stable (see, for instance, [34] and references therein).

We thus modify this strategy, by proposing the following algorithm

1. Take $\left[\begin{array}{l}q_{0} \\ q_{1}\end{array}\right] \in \mathcal{H}_{\frac{3}{2}} \times \mathcal{H}_{\frac{1}{2}}$.

2. For any $h>0$ choose $N(h) \in \mathbb{N}$ as in Theorem 7.1 below. 
3. For $n=1,2, \cdots N(h)$ solve the following coupled systems:

- A forward system

$$
\begin{gathered}
\ddot{w}_{h}^{n}(t)+A_{0 h} w_{h}^{n}(t)+B_{0 h} B_{0 h}^{*} \dot{w}_{h}^{n}(t)=0 \quad(t \geqslant 0) \\
w_{h}^{n}(0)= \begin{cases}\pi_{h} q_{0}, & \text { if } n=1 \\
w_{b, h}^{n-1}(0), & \text { if } 1<n \leqslant N(h)\end{cases} \\
\dot{w}_{h}^{n}(0)= \begin{cases}\pi_{h} q_{1}, & \text { if } n=1 \\
\dot{w}_{b, h}^{n-1}(0), & \text { if } 1<n \leqslant N(h),\end{cases}
\end{gathered}
$$

- A backward system

$$
\begin{gathered}
\ddot{w}_{b, h}^{n}(t)+A_{0 h} w_{b, h}^{n}(t)-B_{0 h} B_{0 h}^{*} \dot{w}_{b, h}^{n}(t)=0 \quad(t \leqslant \tau) \\
w_{b, h}^{n}(\tau)=w_{h}^{n}(\tau), \quad \dot{w}_{b, h}^{n}(\tau)=\dot{w}_{h}^{n}(\tau) .
\end{gathered}
$$

4. Compute $\left[\begin{array}{l}w_{0 h} \\ w_{1 h}\end{array}\right]$ as follows

$$
\left[\begin{array}{l}
w_{0 h} \\
w_{1 h}
\end{array}\right]=\left[\begin{array}{l}
\pi_{h} q_{0} \\
\pi_{h} q_{1}
\end{array}\right]+\sum_{n=1}^{N(h)}\left[\begin{array}{c}
w_{b, h}^{n}(0) \\
\dot{w}_{b, h}^{n}(0)
\end{array}\right]=\sum_{n=1}^{N(h)+1}\left[\begin{array}{c}
w_{h}^{n}(0) \\
\dot{w}_{h}^{n}(0)
\end{array}\right] .
$$

5. Compute the control $u_{h}$,

$$
u_{h}=B_{0 h}^{*} \dot{w}_{h}+B_{0 h}^{*} \dot{w}_{b, h},
$$

where $w_{h}$ and $w_{b, h}$ are the solution of

$$
\begin{gathered}
\ddot{w}_{h}(t)+A_{0 h} w_{h}(t)+B_{0 h} B_{0 h}^{*} \dot{w}_{h}(t)=0 \quad(t \geqslant 0) \\
w_{h}(0)=w_{0 h}, \quad \dot{w}_{h}(0)=w_{1 h}, \\
\ddot{w}_{b, h}(t)+A_{0 h} w_{b, h}(t)-B_{0 h} B_{0 h}^{*} \dot{w}_{b, h}(t)=0 \quad(t \leqslant \tau) \\
w_{b, h}(\tau)=w_{h}(\tau), \quad \dot{w}_{b, h}(\tau)=\dot{w}_{h}(\tau) .
\end{gathered}
$$

We note that a method based on Russell's principle has been used to compute an exact boundary control for a class of second order evolution equations in [26] (see also [12]). With our notation and after discretizing with respect to the space variable, the method in [26] consists in choosing $N(h)=1$. This choice is convenient for implementation purposes but it does not yield the convergence of $u_{h}$ to $u$. In our work the appropriate choice of $N(h)$ plays a central role in order to obtain error estimates. Note also that the above mentioned "natural" methodology consists in taking $N(h)=+\infty$ which is also not providing, in general, the desired convergence and error estimates results.

We can now formulate the main result of this section.

Theorem 7.1. With the above notation and assumptions, assume furthermore that the system (2.2), (2.3) is exactly controllable in some time $\tau>0$ and that $B_{0} B_{0}^{*} \in \mathcal{L}\left(\mathcal{H}_{1}, \mathcal{H}_{\frac{1}{2}}\right)$. Then there exists a constant $m_{\tau}>0$ such that the family $\left(u_{h}\right)_{h>0}$ of $C\left([0, \tau] ; U_{h}\right)$ defined in (7.14) with $N(h)=\left[\theta m_{\tau} \ln \left(h^{-1}\right)\right]$, converges when $h \rightarrow 0$ to an exact control in time $\tau$ of (2.2), (2.3), denoted by $u$, for every $Q_{0}=\left[\begin{array}{l}q_{0} \\ q_{1}\end{array}\right] \in \mathcal{H}_{\frac{3}{2}} \times \mathcal{H}_{1}$. Moreover, there exist constants $h^{*}>0$ and $C:=C_{\tau}$ such that we have

$$
\left\|u-u_{h}\right\|_{C([0, \tau] ; \mathcal{U})} \leqslant C h^{\theta} \ln ^{2}\left(h^{-1}\right)\left\|Q_{0}\right\|_{\mathcal{H}_{\frac{3}{2}}} \times \mathcal{H}_{1} \quad\left(0<h<h^{*}\right) .
$$


We describe below, following [4], the main steps of the proof. To this aim, we need some more notation. For $h>0$ we denote $X_{h}=V_{h} \times V_{h}$ and we consider the operators

$$
\mathcal{A}_{h}=\left[\begin{array}{cc}
0 & I \\
-A_{0 h} & 0
\end{array}\right], \quad \mathcal{B}_{h}=\left[\begin{array}{c}
0 \\
B_{0 h}
\end{array}\right]
$$

The discrete analogues of the semigroups $\mathbb{T}, \mathbb{S}$ and of the operator $L_{t}$ (all introduced in Section 2), denoted by $\mathbb{T}_{h}, \mathbb{S}_{h}$ and $L_{h, t}$ respectively, are defined, for every $h>0$, by

$$
\mathbb{T}_{h, t}=\mathrm{e}^{t\left(\mathcal{A}_{h}-\mathcal{B}_{h} \mathcal{B}_{h}^{*}\right)}, \quad \mathbb{S}_{h, t}=\mathrm{e}^{t\left(-\mathcal{A}_{h}-\mathcal{B}_{h} \mathcal{B}_{h}^{*}\right)}, \quad L_{h, t}=\mathbb{S}_{h, t} \mathbb{T}_{h, t} \quad(t \geqslant 0)
$$

For every $h>0$ we define $\Pi_{h} \in \mathcal{L}\left(\mathcal{H}_{\frac{1}{2}} \times \mathcal{H}_{\frac{1}{2}}, X_{h}\right)$ by

$$
\Pi_{h}=\left[\begin{array}{cc}
\pi_{h} & 0 \\
0 & \pi_{h}
\end{array}\right]
$$

We are now in a position to sketch the proof of main result in this section, and we refer to [4] for the details.

Proof of Theorem 7.1. We first remark that the regularity result in Proposition 2.4 plays an important role. Indeed, as in many problems from the numerical analysis of PDEs systems, such regularity properties of the exact solutions (or control functions in our case) are essential in establishing error estimates. We can thus combine Proposition 2.4 with pretty standard error estimates for evolution equations in Hilbert spaces to obtain that

$$
\left\|\left(u-v_{h}\right)(t)\right\|_{\mathcal{U}} \leqslant \frac{C_{2}+t C_{3}}{1-\left\|L_{\tau}\right\|_{\mathcal{L}\left(\mathcal{H}_{\frac{3}{2}} \times \mathcal{H}_{1}\right)}} h^{\theta}\left\|Q_{0}\right\|_{\mathcal{H}_{\frac{3}{2}} \times \mathcal{H}_{1}} \quad(t \in[0, \tau]),
$$

where $u$ is the exact control (constructed via Russell's principle as in Proposition 2.2) and

$$
v_{h}(t)=\mathcal{B}_{h}^{*} \mathbb{T}_{h, t} \Pi_{h} W_{0}+\mathcal{B}_{h}^{*} \mathbb{S}_{h, \tau-t} \mathbb{T}_{h, \tau} \Pi_{h} W_{0} \quad(t \in[0, \tau])
$$

Moreover, concerning the operators $\left(L_{h}^{t}\right)$ introduced in (7.21), it can be shown that there exist three constants $K_{0}, K_{1}, h^{*}>0$ such that, for every $t \in[0, \tau], h \in\left(0, h^{*}\right)$ and $m \in \mathbb{N}$ we have

$$
\left\|L_{t}^{k} Z_{0}-L_{h, t}^{m} \Pi_{h} Z_{0}\right\|_{X} \leqslant\left(K_{0}+m K_{1} t\right) h^{\theta}\left\|Z_{0}\right\|_{\mathcal{H}_{\frac{3}{2}}} \times \mathcal{H}_{1} \quad\left(Z_{0} \in \mathcal{H}_{\frac{3}{2}} \times \mathcal{H}_{1}\right)
$$

Using the semigroup notation introduced in Section 2 we can write $u_{h}$ given by $(7.14)$ as

$$
u_{h}(t)=\mathcal{B}_{h}^{*} \mathbb{T}_{h, t}\left[\begin{array}{c}
w_{0 h} \\
w_{1 h}
\end{array}\right]+\mathcal{B}_{h}^{*} \mathbb{S}_{h, \tau-t} \mathbb{T}_{h, \tau}\left[\begin{array}{l}
w_{0 h} \\
w_{1 h}
\end{array}\right] \quad(t \in[0, \tau])
$$

where

$$
\left[\begin{array}{l}
w_{0 h} \\
w_{1 h}
\end{array}\right]=\sum_{n=0}^{N(h)} L_{h, \tau}^{n} \Pi_{h}\left[\begin{array}{l}
q_{0} \\
q_{1}
\end{array}\right] .
$$

At this stage we note that the exact control $u$ constructed in Section 2 , where $W_{0}=\left[\begin{array}{l}w_{0} \\ w_{1}\end{array}\right]$ is given by (2.16). 
To prove (7.19) we next remark that

$$
\left\|u-u_{h}\right\|_{C([0, \tau], \mathcal{U})} \leqslant\left\|u-v_{h}\right\|_{C([0, \tau], \mathcal{U})}+\left\|v_{h}-u_{h}\right\|_{C([0, \tau], \mathcal{U})},
$$

so it suffices to evaluate the two terms from the right, where $v_{h}$ is given by (7.24).

To estimate the second term in the right-hand side of (7.28) we first note that

$$
\begin{aligned}
& \left(v_{h}-u_{h}\right)(t) \\
& \quad=\mathcal{B}_{h}^{*} \mathbb{T}_{h, t} \Pi_{h} W_{0}+\mathcal{B}_{h}^{*} \mathbb{S}_{h, \tau-t} \mathbb{T}_{h, \tau} \Pi_{h} W_{0}-\mathcal{B}_{h}^{*} \mathbb{T}_{h, t} \Pi_{h}\left[\begin{array}{c}
w_{0 h} \\
w_{1 h}
\end{array}\right]-\mathcal{B}_{h}^{*} \mathbb{S}_{h, \tau-t} \mathbb{T}_{h, \tau} \Pi_{h}\left[\begin{array}{c}
w_{0 h} \\
w_{1 h}
\end{array}\right] .
\end{aligned}
$$

It follows that there exists a positive constant $C$ such that, for any $t \in[0, \tau]$,

$$
\begin{aligned}
&\left\|\left(v_{h}-u_{h}\right)(t)\right\|_{\mathcal{U}} \leqslant\left\|\mathcal{B}_{h}^{*} \mathbb{T}_{h, t} \Pi_{h} W_{0}-\mathcal{B}_{h}^{*} \mathbb{T}_{h, t} \Pi_{h}\left[\begin{array}{c}
w_{0 h} \\
w_{1 h}
\end{array}\right]\right\|_{\mathcal{U}} \\
&+\left\|\mathcal{B}_{h}^{*} \mathbb{S}_{h, \tau-t} \mathbb{T}_{h, \tau} \Pi_{h} W_{0}-\mathcal{B}_{h}^{*} \mathbb{S}_{h, \tau-t} \mathbb{T}_{h, \tau} \Pi_{h}\left[\begin{array}{c}
w_{0 h} \\
w_{1 h}
\end{array}\right]\right\|_{\mathcal{U}} \\
& \leqslant C\left\|W_{0}-\left[\begin{array}{c}
w_{0 h} \\
w_{1 h}
\end{array}\right]\right\|_{X}=C\left\|\sum_{n=0}^{\infty} L_{\tau}^{n} Q_{0}-\sum_{n=0}^{N(h)} L_{h, \tau}^{n} \Pi_{h} Q_{0}\right\|_{X} \\
& \leqslant C \sum_{n=N(h)+1}^{\infty}\left\|L_{\tau}\right\|_{\mathcal{L}(X)}^{n}\left\|Q_{0}\right\|_{X}+C \sum_{n=0}^{N(h)}\left\|\left(L_{\tau}^{n}-L_{h, \tau}^{n} \Pi_{h}\right) Q_{0}\right\|_{X} .
\end{aligned}
$$

The above estimate and (7.25) imply that there exists $\widetilde{C}>0$ such that

$$
\begin{aligned}
\left\|v_{h}-u_{h}\right\|_{C([0, \tau] ; \mathcal{U})} & \leqslant C \frac{\left\|L_{\tau}\right\|_{\mathcal{L}(X)}^{N(h)+1}}{1-\left\|L_{\tau}\right\|_{\mathcal{L}(X)}}\left\|Q_{0}\right\|_{X}+C h^{\theta} \sum_{n=0}^{N(h)}\left(C_{0}+n C_{1} \tau\right)\left\|Q_{0}\right\|_{\mathcal{H}_{\frac{3}{2}} \times \mathcal{H}_{1}} \\
& =C \frac{\left\|L_{\tau}\right\|_{\mathcal{L}(X)}^{N(h)+1}}{1-\left\|L_{\tau}\right\|_{\mathcal{L}(X)}}\left\|Q_{0}\right\|_{X}+C(N(h)+1)\left(C_{0}+C_{1} \frac{N(h)}{2} \tau\right) h^{\theta}\left\|Q_{0}\right\|_{\mathcal{H}_{\frac{3}{2}}} \times \mathcal{H}_{1} \\
& \leqslant C \frac{\left\|L_{\tau}\right\|_{\mathcal{L}(X)}^{N(h)+1}}{1-\left\|L_{\tau}\right\|_{\mathcal{L}(X)}}\left\|Q_{0}\right\|_{X}+\widetilde{C} N^{2}(h)(1+\tau) h^{\theta}\left\|Q_{0}\right\|_{\mathcal{H}_{\frac{3}{2}}} \times \mathcal{H}_{1} \\
& \leqslant \frac{\widetilde{C}(1+\tau)}{1-\left\|L_{\tau}\right\|_{\mathcal{L}(X)}}\left(\left\|L_{\tau}\right\|_{\mathcal{L}(X)}^{N(h)}+N^{2}(h) h^{\theta}\right)\left\|Q_{0}\right\|_{\mathcal{H}_{\frac{3}{2}}} \times \mathcal{H}_{1} .
\end{aligned}
$$

Choosing $N(h)=\left[\frac{\theta}{\ln \left(\left\|L_{\tau}\right\|_{\mathcal{L}(X))}\right.} \ln (h)\right]$ we deduce that

$$
\left\|v_{h}-u_{h}\right\|_{C([0, \tau] ; U)} \leqslant \frac{\widetilde{C}(1+\tau)}{\left(1-\left\|L_{\tau}\right\|_{\mathcal{L}(X)}\right) \ln ^{2}\left(\left\|L_{\tau}\right\|_{\mathcal{L}(X)}^{-1}\right)} \ln ^{2}\left(h^{-1}\right) h^{\theta}\left\|Q_{0}\right\|_{\mathcal{H}_{\frac{3}{2}}} \times \mathcal{H}_{1} .
$$

Combining this last estimate with $(7.23)$ and taking $m_{\tau}=\frac{1}{\ln \left(\left\|L_{\tau}\right\|_{\mathcal{L}(X)}^{-1}\right)}$ we obtain the conclusion (7.19). 


\section{References}

[1] K. Ammari And M. Tucsnak, Stabilization of second order evolution equations by a class of unbounded feedbacks, ESAIM Control Optim. Calc. Var., 6 (2001), pp. 361386 (electronic).

[2] C. Bardos, G. Lebeau, And J. Rauch, Sharp sufficient conditions for the observation, control, and stabilization of waves from the boundary, SIAM J. Control Optim., 30 (1992), pp. 1024-1065.

[3] G. Chen, Control and stabilization for the wave equation in a bounded domain, SIAM J. Control Optim., 17 (1979), pp. 66-81.

[4] N. Cîndea, S. Micu, And M. Tucsnak, An approximation method for exact controls of vibrating systems, SIAM J. Control Optim., 49 (2011), pp. 1283-1305.

[5] B. Dehman and G. LeBeau, Analysis of the HUM control operator and exact controllability for semilinear waves in uniform time, SIAM J. Control Optim., 48 (2009), pp. 521-550.

[6] G. Doetsch, Introduction to the theory and application of the Laplace transformation, Springer-Verlag, New York-Heidelberg, 1974. Translated from the second German edition by Walter Nader.

[7] S. Ervedoza And E. Zuazua, Hilbert uniqueness method and regularity: Applications to the order of convergence of discrete controls for the wave equation, (2009). Private communication.

[8] S. Ervedoza And E. ZuazuA, Uniformly exponentially stable approximations for a class of damped systems, J. Math. Pures Appl. (9), 91 (2009), pp. 20-48.

[9] C. FABRe, Exact boundary controllability of the wave equation as the limit of internal controllability, SIAM J. Control Optim., 30 (1992), pp. 1066-1086.

[10] C. Fabre And J.-P. Puel, Behavior near the boundary for solutions of the wave equation, J. Differential Equations, 106 (1993), pp. 186-213.

[11] _ Pointwise controllability as limit of internal controllability for the wave equation in one space dimension, Portugal. Math., 51 (1994), pp. 335-350.

[12] R. Font And F. Periago, Numerical simulation of the boundary exact control for the system of linear elasticity, Applied Mathematics Letters, 23 (2010), pp. 1021 1026 .

[13] R. Glowinski, C. H. Li, And J.-L. Lions, A numerical approach to the exact boundary controllability of the wave equation (i). dirichlet controls: Description of the numerical methods, Japan J. Appl. Math., 7 (1990), pp. 1-76.

[14] R. Glowinski And J.-L. Lions, Exact and approximate controllability for distributed parameter systems, Acta Numer., (1996), pp. 159-333.

[15] A. Haraux, Une remarque sur la stabilisation de certains systèmes du deuxième ordre en temps, Portugal. Math, 46 (1989), pp. 245-258. 
[16] K. Ito, K. Ramdani, And M. TuCsnak, A time reversal based algorithm for solving initial data inverse problems, Discrete Contin. Dyn. Syst. Ser. S., (2010). to appear.

[17] K. Ito, K. Ramdani, And M. Tucsnak, A time reversal based algorithm for solving initial data inverse problems, Discrete and Continuous Dynamical Systems-Series S, 4 (2011), pp. 641-652.

[18] _ - A time reversal based algorithm for solving initial data inverse problems, Discrete Contin. Dyn. Syst. Ser. S, 4 (2011), pp. 641-652.

[19] R. Joly, Convergence of the wave equation damped on the interior to the one damped on the boundary, J. Differential Equations, 229 (2006), pp. 588-653.

[20] V. Komornik, Exact controllability and stabilization, RAM: Research in Applied Mathematics, Masson, Paris; John Wiley \& Sons, Ltd., Chichester, 1994. The multiplier method.

[21] G. Lebeau And M. Nodet, Experimental study of the HUM control operator for linear waves, Experiment. Math., 19 (2010), pp. 93-120.

[22] J.-L. Lions, Contrôlabilité exacte, perturbations et stabilisation de systèmes distribués. Tome 1, vol. 8 of Recherches en Mathématiques Appliquées, Masson, Paris, 1988. With a chapter by E. Zuazua, and a chapter by C. Bardos, G. Lebeau and J. Rauch.

[23] K. LiU, Locally distributed control and damping for the conservative systems, SIAM J. Control Optim., 35 (1997), pp. 1574-1590.

[24] V. Natarajan And G. Weiss, Improving the exponential decay rate by back and forth iterations of the feedback in time, in Decision and Control (CDC), 2013 IEEE 52nd Annual Conference on, IEEE, 2013, pp. 2715-2719.

[25] A. PAZY, Semigroups of linear operators and applications to partial differential equations, vol. 44 of Applied Mathematical Sciences, Springer-Verlag, New York, 1983.

[26] P. Pedregal, F. Periago, and J. Villena, A numerical method of local energy decay for the boundary controllability of time-reversible distributed parameter systems, Stud. Appl. Math., 121 (2008), pp. 27-47.

[27] K. Ramdani, M. Tucsnak, and G. Weiss, Recovering the initial state of an infinite-dimensional system using observers, Automatica, 46 (2010), pp. 1616-1625.

[28] R. Rebarber and G. Weiss, An extension of Russell's principle on exact controllability, in Proc. of the Fourth ECC, 1997. CD-ROM.

[29] D. Russell, Controllability and stabilizability theory for linear partial differential equations: recent progress and open questions, SIAM Rev., 20 (1978), pp. 639-739.

[30] H. Shim, A. TAnwani, And Z. PIng, Back-and-forth operation of state observers and norm estimation of estimation error, in Decision and Control (CDC), 2012 IEEE 51st Annual Conference on, IEEE, 2012, pp. 3221-3226. 
[31] M. Tucsnak and G. Weiss, How to get a conservative well-posed linear system out of thin air. Part II: Controllability and stability, SIAM J. Control Optimization, 42 (2003), pp. 907-935.

[32] M. TuCsnak AND G. Weiss, Observation and control for operator semigroups, Birkhäuser Advanced Texts: Basler Lehrbücher. [Birkhäuser Advanced Texts: Basel Textbooks], Birkhäuser Verlag, Basel, 2009.

[33] G. Weiss And M. Tucsnak, How to get a conservative well-posed linear system out of thin air. Part I: Well-posedness and energy balance, ESAIM Control Optim. Calc. Var., 9 (2003), pp. 247-274.

[34] E. ZuazuA, Propagation, observation and control of waves approximated by finite difference methods, SIAM Rev., 47 (2005), pp. 197-243. 